\title{
Zur Frage von der Behandlung mit Quecksilber-Säckchen.
}

\author{
Von \\ Professor Edvard Welander \\ in Stockholm.
}

Im Archiv für Dermatologie und Syphilis, Band LI, hat Schuster einen Aufsatz: "Zur Behandlung mittels Quecksilbersäckchen und Mercolint" veröffentlicht, in welchem er sagt: „Die uns hier und 1. c. interessirende Hauptfrage bleibt die, ob die Welander'sche Säckchenbehandlung, oder die an Stelle des Sackes von Blaschko (s. Berliner Kl. Wochenschr., Nr. 46, Nov.) eingefiuhrte Mercolintbehandlung, d. h. die mittelst Baumwollenlappen oder Schurze, welche in gleichmässiger Weise mit $\mathrm{Hg}$ imprägnirt und von Beiersdorf-Hamburg zu beziehen sind, ob diese ein ausreichender Ersatz der bis jetzt üblichen Einreibecur ist oder zu werden verspricht?"

Anlässlich theils dieses, theils ein paar anderer Aufsätze, in denen über das mit der Behandlung mit Quecksilbersäckchen erhaltene Ergebniss berichtet wird, möchte ich gern einige Worte äussern, erstens in Betreff der Schlüsse, die hinsichtlich des therapeutischen Werthes der Methode gezogen worden sind, zweitens vor allem in Betreff der Frage von der Absorption des Quecksilbers, denn neue Untersuchungen haben mich uiberzeugt, dass, wie ich angegeben, bei der Behandlung mit Hg-Säckchen eine schnelle und kräftige Absorption von $\mathrm{Hg}$ stattfindet und nach der Behandlung $\mathrm{Hg}$ lange und kräftig im Organismus remanirt, wogegen die Ansicht, der ich hinsicht- 
lich des Weges der Absorption des Quecksilbers am meisten zugeneigt habe, nämlich duss dieselbe zum allergrössten Theil durch die Respirationsorgane geschieht, nicht ganz richtig ist, da, wie $\mathrm{Piccardi}$ gezeigt hat, $\mathrm{Hg}$ auch durch die Haut absorbirt wird, jedoch, wie von mir stets hervorgehoben worden ist, kein eingeriebenes, sondern nur verdunstetes, d. h. in Dämpfe aufgelöstes.

In den Wiener Med. Blättera vom 29. März 1900 theilt Gschwend mit, dass er in Jarisch's Abtheilung in Graz eine grosse Anzahl Versuche mit diesen $\mathrm{Hg}$-Säckchen angestellt habe, von welchen Versuchen $46 \mathrm{ganz}$, wie ich es vorgeschrieben und 16 mit einer Abänderung meiner Vorschriften ausgeführt worden sind. Er hat diese Fälle nicht ausgewählt, was daraus zu ersehen ist, dass von ihnen 28 maculöse, 22 lenticuläre, 5 kleinpapulöse (Lichen syph.), 5 pustulöse und 2 Syphilide ohne unirersales Exanthem waren. Von dem therapeutischen Ergebnisse sagt er: „Bezüglich der Wirkungsweise der Quecksilbersäckchen ergab sich das erfreuliche Resultat, dass bis zum Verschwinden des Exanthems mittelst der Welander'schen Methode durchschnittlich nur 14 Säckchen und mittelst der modificirten Behandlung 'bloss 15 Säckchen nothwendig waren, wäbrend ich z. B. bei einer Zusammenstellung von 50 beliebigen Krankengeschichten des Jahres 1898 aus der Grazer dermatologischen Klinik gefunden, dass diesbezüglich 16 Einreibungen erforderlich waren." Dieses ist ja ein sehr günstiges Ergebniss, und dasselbe steht ganz mit dem von mir in Hunderten von Fällen gewonnenen in Uebereinstimmung.

Aber, sagt G schwend, die Methode hat doch, ungeachtet ihrer Verdienste: Einfachheit und Reinlichkeit, ihre Ungelegenheiten, und diese sind: oft auftretende Stomatitis und schnell eintretende Recidive.

Gschwend hat Stomatitis in 35 Proc. der mit HgSäckchen, aber nur in 14 Proc. der mit Einreibungen behandeinden Fälle auftreten sehen. Hierbei will ich auf das Eigenthü oliche hinweisen, dass sowohl in meiner privaten Praxis, wie auch im Krankenhause St. Göran sehr selten Fälle ron Stomatitis rorgekommen sind, ja so selten, dass ich bei den Vorlesungen im Krankenhause oft wochenlang keinen Fall von 
Stomatitis habe vorzeigen können. Da wir nun faktisch so wenig von der Ursache der mercurialen Stomatitis wissen, will ich hier durchaus nicht versuchen, eine hypothetische Erklärung ihres so verschiedenen Auftretens in Graz und an anderen Stellen und im Krankenhause St. Göran zu geben. Ich will nur auf einige Worte hinweisen, die ich in dieser Frage in einem Aufsatz: "Einige Worte über die Mercolintschürze," in der Festschrift für Herrn Hofrath $\mathrm{J}$. $\mathrm{Ne}$ u m a n n geäussert habe.

Eine viel wichtigere Anmerkung von $\mathrm{Gsch}$ wend ist die, dass Recidive so früh auftreten, weshalb ich auch etwas näher darauf eingehen will.

Es ist äusserst schwer, auf Grund der auftretenden Recidive zu entscheiden, welche Methode vor anderen den Vorzug verdient, und es ist nothwendig, dass man als Stïtze für ein solches Ürtheil Fälle wählt, die man Gelegenheit gehabt hat, lange zu verfolgen und genau zu beobachten. Welche Behandlungsmethode man auch anwenden mag, so sieht man in den meisten Fällen, wenn man sie lange und genan zu beobachten Gelegenheit hat, Recidive oder doch wenigstens das eine oder andere Symptom auftreten, sofern nämlich die Krankheit sich in einem frühen Stadium befindet. Wenn man nun Gelegenheit hat, alle Fälle einer Untersuchungsserie zu verfolgen, so kann die Angabe über die eingetretenen Recidive selbstredend eine ganz andere werden, als wenn man genöthigt ist, seine Berechnungen in dieser Hinsicht nach einigen Fällen zu machen, die man zufällig in der Lage ist verfolgen $\mathbf{z u}$ können. Vergleicht man zwei Behandlungsmethoden mit einander und hat man Gelegenheit, je 20 Procent der Fälle, in denen man sie zur Anwendung bringt, zu verfolgen, so kann es der Zufall fügen, dass von der einen Serie einige Fälle von früh auftretendem Recidiv aufgezeichnet werden, wäbrend man von der anderen keinen solchen Fall zu sehen bekommt, obschon frühe Recidive auch hier, und vielleicht sogar in grösserer Anzahl als auf der anderen Seite eintreffen können. Sollte man nun nach diesen wenigen Fällen, die man zufälligerweise beobachten kann, urtheilen und von der einen Methode sagen wollen, dass bei ihrer Anwendung oft trühe Recidive eintreten, und ron der andern, dass bei ihr nie, oder beinahe 
nie frühe Recidive vorkommen, so würde man ja ein ganz falsches Urtheil fällen, und noch unrichtiger würde es sein, daraus den Schluss zu ziehen, dass bei Anwendung der einen Methode $\mathrm{Hg}$ nur eine kurze, bei Anwendung der anderen eine lange Zeit im. Organismus remanirt. Zur Anstellung eines Vergleiches zwischen zwei Methoden in dieser Hinsicht tangen nar Serien, wo a 11 e Fälle haben beobachtet werden können.

Aber nun haben wir uns darüber klar zu werden, was unter einem Recidiv zu verstehen ist. Zuweilen, namentlich in einem frühen Stadium der Krankheit, können wir ja bei einer Person, die viel raucht, eine geringe, unbedeutende Syphilide érosive im Munde, oder bei einer Person, die sich nicht rein hält, ein solches unbedeutendes Syphilid in der Ano-Genitalgegend auftreten sehen, und dieses sehr bald nach der kräftigsten Hg-Behandlung, iwelcher Art sie auch gewesen sein mag, und ungeachtet sich nachweisen lässt, dass eine höchst bedeutende Menge $\mathrm{Hg}$ im Organismus remanirt. Ich könnte eine Menge solche bei Anwendung verschiedener Behandlungsmethoden und gleich nach einer grossen Anzahl Einspritzungen von unlöslichen $\mathrm{Hg}$-Salzen aufgetretene Fälle anführen. Mit Ausnahme dieses geringen Symptomes, das bei der einfachsten localen Behandlung in der Regel sehr bald wieder verschwindet, sehen wir oft während langer Zeit kein einziges anderes Symptom, am allerwenigsten aber einen allgemeinen Ausbruch der Syphilis auftreten. Eine solche geringfügige Syphilide érosive oder entsprechendes Symptom ist, scheint es mir, nicht als ein wirkliches Recidiv aufzufassen; das Auftreten eines solchen Symptomes liefert auch nicht den geringsten Beweis dafür, dass eine Behandlung nicht kräftig und wirksam gewesen ist oder dass nicht eine grosse Menge $\mathrm{Hg}$ im Körper remanirt und für lange Zeit gegen ein wirkliches Recidiv schiitzt. Wollen wir nun eine solche kleine Hautlosigkeit als ein Recidiv auffassen, so müssen wir zu gleicher Zeit zugestehen, dass wir keine Hg-Behandlungsmethode besitzen, die kräftig genug ist, um wenigstens in der allernächsten Zeit nach Schluss der Behandlung das Auftreten von Recidiven verhindern zu können. Als Recidive scheinen mir in einem frühen Stadium der Krankheit mit Grund nur die Fälle bezeichnet werden zu können, 
wo ein allgemeiner Ausbruch der Syphilis eintritt oder wo sich (am liebsten) an mehreren Stellen, wie z. B. an verschiedenen Schleimhäuten, mehrere Efflorescenzen (mucöse Papeln) unabhängig von offenbar localer Reizung zeigen.

Solche wirklichen Recidive treten nun in den allermeisten Fällen erst einige Wochen oder noch längere Zeit nach einer kräftigen Hg-Behandlung auf, d. h. erst nachdem ein grosser Theil des absorbirten Quecksilbers eliminirt worden ist; aber solche Recidive habe ich doch, obschon nur in äusserst seltenen Fällen, ziemlich bald nach der kräftigsten $\mathrm{Hg}$-Behandlung auftreten sehen, und dieses in Fällen, die nicht als Syphilis maligna aufgefasst werden können. Weshalb nun diese Recidive aufgetreten sind, habe ich nicht erklären können, aber deshalb, weil sie aufgetreten sind, habe ich mich gleichwohl nicht für berechtigt angesehen, zu erklären, dass die angewendete Behandlungsmethode, z. B. Injection von unlöslichen $\mathrm{Hg}$-Salzen, keine kräftige Behandlung ist, noch weniger den Schluss zu ziehen, dass bei Anwendung dieser Methode $\mathrm{Hg}$ nicht lange im Organismus remanirt.

Nun habe ich auch bei der Anwendung der Hg-Säckchenmethode ein paarmal ein wirkliches Recidiv ziemlich bald nach der Behandlung auftreten sehen, ohne dass ich deshalb geglaubt habe berechtigt zu sein, diese Methode gering zu schätzen; denn es ist bei dieser, wie bei allen anderen Methoden, der Verlauf der grossen Mehrzahl der Fälle, den man in Betracht zu ziehen hat.

Weiter muss man natïrlicherweise bei dieser gleich wie bei jeder anderen Behandlungsmethode Rücksicht auf die Zeit nehmen, welche der Patient behandelt worden ist. Ist er nur 2-3 Wochen behandelt worden, so ist es ja klar, dass $\mathrm{Hg}$ nicht in derselben Menge und gleich lange im Organismus remaniren kann, wie wenn er 5-6 Wochen behandelt worden ist, und dass man also im ersten Falle Schutz gegen Recidive nicht für so lange Zeit erwarten kann, wie man im letzten Falle zu hoffen das Recht hat.

Aber wir müssen auch die Form der Symptome in Betracht ziehen, die der Patient gehabt hat; nach schwereren Formen sehen wir ja oft Recidive eher eintreten als nach leichteren. 
Schliesslich haben wir auch die Form zu berücksichtigen, unter welcher das Recidiv auftritt. Zeigt es sich, dass bei Anwendung einer Behandlungsmethode Recidive oft unter schwereren Formen als der vorhergegangene Ausbruch der Krankheit auftreten, so deutet dieses darauf hin, dass die Methode nicht hinreichend kräftig ist, n. b. wenn nicht ein langer Zeitraum nach der Behandlung verflossen ist.

Ich habe dieses hier hervorheben wollen, um zu zeigen, wie wichtig es ist, die verschiedenen Verhältnisse beim Auftreten eines Recidives genau zu beurtheilen, wenn man auf Grund desselben entscheiden will, ob eine Methode einen Vorzug vor einer anderen hat.

Prüfen wir nun Gsch wend's Angaben über Recidive bei der Behandlung mit Hg-Säckchen, so finden wir, dass er solche in 20 Procent seiner Fälle gesehen hat, was, wie er auch selbst sagt, ganz sicher zu wenig ist, denn wenigstens in den frischen Fällen von Syphilis ist bei dieser Methode, was gegebener Weise auch bei jeder anderen Methode eingetroffen sein würde, das Procent der Recidive bedeutend grösser gewesen, obschon Gschwend davon keine Kenntniss erhalten hat.

Es hätte von Interesse sein können, zu erfahren, in wie viel Procent der Fälle bei Anwendung der wirklichen Einreibungscur Recidive aufgetreten sind, sowie auch, wie bald nach der Behandlung sie sich gezeigt haben. Gschwend hat einen interessanten Vergleich zwischen dem Zeitpunkt des Verschwindens der Symptome bei diesen beiden Behandlungsformen angestellt; von Interesse würde es auch gewesen sein, wenn er einen Vergleich zwischen dem Zeitpunkte des Auftretens der Recidive bei ihnen angestellt hätte, obschon ich willig zugebe, dass dieser Vergleich keinen wirklichen Werth gehabt haben könnte, da die Recidive vermuthlich auch bei der Einreibungscur nur von einer geringeren Zahl der Fälle bekannt sind.

Von besonders grossem Interesse würde es gewesen sein zu erfahren, unter welcher Form die Recidive bei der Behandlung mit $\mathrm{Hg}$-Säckchen aufgetreten sind, ob es nur ein einzelnes, unbedeutendes Symptom, oder ob es ein allgemeiner 
Ausbruch der Syphilis gewesen ist; leider ist hierüber nicht die geringste Angabe gemacht worden.

Gs chwend sagt, dass die Recidive sehr bald aufgetreten sind; zwei haben sich schon 8 Tage nach der Entlassung aus der Spitalsbehandlung, zwei andere nach 14 Tagen, drei nach 3-4 Wochen, zwei nach 6 Wochen und die übrigen nach $2-3^{1 / 2}$ Monaten gezeigt. Was die Recidive anbelangt, die 2-31/2 Monate nach der Beendigung der Behandlung (denn ich vermuthe, dass dieselbe mit der Entlassung aus der Spitalsbehandlung zusamenfällt) aufgetreten sind, so lassen sich diese Recidive wohl nicht mit Recht als früh aufgetreten bezeichnen, und dieses dürfte auch von den Recidiven gelten können, die sich 6 Wochen nach der Behandlung gezeigt haben, denn solche- sind wohl bei Anwendung jeder beliebigen Behandlungsform beobachtet worden.

Die Anmerkung in Betreff früh auftretender Recidive kann also eigentlich nur den übrigen Fällen gelten. Dass es namentlich in diesen von dem grössten Interesse gewesen wäre zu wissen, unter welcher Form die Recidive aufgetreten, ob es wirkliche Recidive oder nur einzelne Symptome gewesen sind, ist ja selbstverständlich; leider sagt $G \mathrm{schwend}$ nichts darüber. Ich habe indessen in folgendem Verzeichniss sämmtliche Recidive zusammengestellt und dabei Rücksicht theils auf die Symptome während der nächstvorhergehenden Behandlung, theils auf die Anzahl der Behandlungstage und theils auf die Anzahl der Tage genommen, die zwischen der vorhergehenden Behandlung und dem Auftreten des Recidives verflossen sind

Recidive bei der Behandlung mit Quecksilbersäckchen (Gschwend).

\begin{tabular}{|c|c|c|c|}
\hline Frische Syphilis & Form des Syphilides & $\begin{array}{c}\text { Anzahl der } \\
\text { Behandlungs- } \\
\text { tage }\end{array}$ & $\begin{array}{l}\text { Recidiv, auf- } \\
\text { getreten nach } \\
\text { der Behandlung }\end{array}$ \\
\hline Frische Syphilis & Grosspapulöses Syphilid & 17 Tage & 8 Tage \\
\hline$\eta$ & Roseola & 22 & 8 \\
\hline \# & Grosspapulöses Syphilid & 19 & 14 \\
\hline
\end{tabular}

Arch. f. Dermat. u. Syph. Bd. LIV. 


\begin{tabular}{|c|c|c|c|}
\hline $\begin{array}{c}\text { Frisehe Syphilis oder } \\
\text { Recidiv }\end{array}$ & Form des Syphilides & $\begin{array}{c}\text { Anzahl der } \\
\text { Behandlungs- } \\
\text { tage }\end{array}$ & \begin{tabular}{|c} 
Recidiv, auf- \\
getreten naeb \\
der Behandlung
\end{tabular} \\
\hline Frische Syphilis & Grosspapulöses Syphilid & 28 Tage & 14 Tage \\
\hline$n$ & $n$ & 17 & 3 Wochen \\
\hline Recidiv & $\pi$ & 28 & 4 \\
\hline Frische Syphilis & Pustulöses Syphilid & 26 & 4 \\
\hline Recidiv & Roseola & 20 & 6 \\
\hline$"$ & $\pi$ & 27 & $6 \quad "$ \\
\hline$\eta$ & Grosspapulöses Syphilid & 18 & 2 Monate \\
\hline Frische Syphilis & $n$ & 33 & 2 \\
\hline$n$ & Roseola & 31 & $2^{1 / 2}$ Monate \\
\hline$n$ & $n$ & 21 & $2 \%$ \\
\hline$n$ & Grosspapulöses Syphilid & 19 & 3 Monate \\
\hline$n$ & $"$ & 16 & $3 \frac{1}{2}$ Monate \\
\hline
\end{tabular}

Beim ersten Blick auf dieses Verzeichniss fällt die unbedeutende Anzahl der Behandlungstage in die Augen, sowohl im allgemeinen, wie besonders in den beiden Fällen, wo das Recidir schon 8 Tage nach der Behandlung aufgetreten und die Zahl der Behandlungstage, ungeachtet diese Fälle frische Syphilis waren, in dem einen Falle 22 und in dem anderen 17 gewesen sind. Dieselbe Anmerkung ist in Betreff zweier Fälle von grosspapulösem ersten Syphilid zu machen, wo das Recidiv in dem einen Falle 14 Tage und in dem anderen 3 Wochen 
nach der Behandlung aufgetreten ist und der Patient in dem ersteren Falle 19 und in dem anderen nur 17 Tage behandelt worden war. Man kann ja, namentlich bei einem ersten Ausbruch der Syphilis unter der Form eines papulösen Syphilides, nicht einmal hoffen, dass eine so kurze Behandlung, von welcher Form dieselbe auch sein möge, für lange Zeit vor einem Recidive zu schützen vermöge; man dürfte dieses also auch nicht von der Hg-Säckchenmethode fordern können.

Was die übrigen Fälle betrifft, sei es, dass Recidive aufgetreten sind oder nicht, so muss ich die Anmerkung machen, dass sie allzu kurze Zeit in Behandlung gewesen sind, was die folgende kleine Zusammenstellung der Fälle von frischer Syphilis deutlich zeigen dürfte.

In einem Falle währte die Behandlung 5 Tage, in zwei Fällen 11-15 Tage, in zehn Fällen 16-20 Tage, in zwölf Fällen 21-25 Tage, in elf Fällen 26- 30 Tage und in sieben Fällen über 30 Tage. Wir finden also, dass von allen Fällen von frischer Syphilis, viele von ihnen mit papulösem und vier mit pustulösem Syphilid, nur 16 Procent länger als 30 Tage behandelt worden sind; 58.1 Procent sind weniger als 25 Tage behandelt worden, ja! bei 30 Procent hat die erste Behandlung der frischen Syphilis weniger als 20 Tage gewährt. Man ist nicht berechtigt, nach einer so kurzen Behandlung zu erwarten, dass Recidive eine längere Zeit ausbleiben werden.

Indessen zieht nun $\mathrm{Gschwend}$ daraus, dass Recidive früh nach der Behandlung aufgetreten sind, den Schluss, dass bei der Behandlung mit Hg-Säckchen $\mathrm{Hg}$ nur kurze Zeit im Organismus remanirt; aber er könnte mit ebenso gutem Recht einen ganz entgegengesetzten Schluss gezogen haben; er hätte sich ebenso gut an die Fälle halten können, wo die Recidive spät aufgetreten sind, wie an die, wo sie sich sehr früh eingestellt haben, denn die Anzahl dieser Recidive ist ja so gering wie 3-4 von jeder Art. Er könnte ja wie folgt raisonnirt haben : trotz einer so kurzen Behandlungszeit wie 16, 19 und 21 Tage hat es gleichwohl $2^{1 / 2}-3$ Monate gedauert, bis ein Recidiv aufgetreten ist; in Fällen, wo die Behandlungszeit so kurz wie $5,13,14,15$ und 17 Tage gewesen ist, hat sich kein Recidiv gezeigt; dieses beweist mit grosser Wahrscheinlichkeit, 
dass bei der Behandlung mit Hg-Säckchen Hg lange im Organismus remanirt und ihn lange vor Recidiven schïtzt - aber natürlicherweise wäre der eine wie der andere Schluss vollkommen unrichtig. Die Frage von der Remanenz des Quecksilbers wird nicht durch solche Raisonnements gelöst; dieses kann nur durch wirkliche $\mathrm{Hg}$-Unternehmungen geschehen (siehe über solche weiter hinten).

Nachdem Gschwend über die Ungelegenheiten der Methode berichtet hat, frägt er: "Können wir sie vervollkommnen?" Fr gibt selbst die Antwort: "die zunächst gelegene Idee einer Verbesserung ist die länger dauernde Application des Quecksilbersäckchens", obschon er diese selbstverständliche Antwort auf die Frage nicht acceptirt. Dieser Vorschlag zu einer Vervollkommnung bedeutet zwar keine Verbesserung der Methode, aber eine bessere praktische Anwendung derselben in Uebereinstimmung mit den von mir aufgestellten Principien und auch mit den Ansichten, die bei allen anderen Hg-Behandlungsmethoden zur Geltung kommen. Weshalb soll bei der Hg-Säckchenmethode die Behandlungszeit kürzer als bei allen anderen Methoden, namentlich der Einreibungscur sein, und dies insonderheit in Fällen von frischer Syphilis ?

Eine Verbesserung würde dann ausserdem die sein, nicht erst $2 \frac{1}{2}-3 \frac{1}{2}$ Monate auf das Auftreten von einem Recidiv zu warten, sondern, wie Fournier, Neisser n. A. gethan haben, die Fälle intermittent preventiv zu behandeln. Für eine solche Behandlung eignet sich die Hg-Säckchenmethode auf Grund ihrer Einfachheit und Reinlichkeit sehr gut. Für meinen Theil bin ich mit den Ergebnissen, die ich mit einer solchen Behandlungsweise der Syphilis erhalten habe, äusserst zufrieden.

Ich will hier hinzufügen, dass alles, was ich über die Behandlung mittelst $\mathrm{Hg}$-Säckchen mit Ung. Hydr. geäussert habe, in der Hauptsache auch auf die Säckchenbehandlung mit Mercuriol seine Anwendung hat und sie auch auf die Behandlung mit der Mercolintschürze, wenn einige Veränderungen in ihrer Grösse, Applicirung u. s. w. vorgenommen werden (siehe meinen Aufsatz über die Mercolintschürze), bekommen wird. 
Wolfsohn hat die Methode in 66 Fällen mit Vortheil angewendet; Siebert hat sie gegen Syphilis bei zarten Kindern zur Anwendung gebracht und dabei (wie ich) gefunden, dass sie in solchen Fällen gute Dienste thut.

Schliesslich hat Jordan theils in einem Aufsatze im Archiv f. Dermat. u. Syph., theils in einem Aufsatz in der Monatsschr. f. prakt. Dermat. seine in Bezug auf diese Methode gewonnenen Erfahrungen mitgetheilt. In 18 Fällen hat er mit ihr ein sehr gutes Resultat erzielt; in 12 Fällen ist das gewonnene Resultat unbefriedigend gewesen. Er frägt sich nun, was dieses unbefriedigende Resultat in diesen 12 Fällen veranlasst haben kann und glaubt, dass die Ursache desselben theils in der Schwere der Krankheitsfälle, theils auch darin $\mathbf{z u}$ sehen sei, dass die Patienten es nicht richtig verstanden hatten, das Säckchen zu tragen. Er empfiehlt die Methode fuir intelligente, breit gebaute Kranke. $\mathrm{R}$ oth hat auch einige Versuche mit dieser Behandlungsmethode gemacht und darzuthun gesucht, dass Quecksilber durch die Haut absorbirt wird.

Wir kommen nun zu der zweiten Abtheilung dieses Aufsatzes, nämlich der Absorption des Quecksilbers bei Anwendung der auf seine Verdünstung basirten Behandlungsmethoden. Glücklicherweise scheint es nunmehr, als ob die Forscher zugäben, dass bei Anwendung dieser Methoden wirklich Quecksilber rom Organismus absorbirt wird, und ich fand mit der grössten Befriedigung, dass S chuster in seinem letzten Aufsatze dieses nicht nur zugesteht, sondern geradezu eine auf dieses Princip basirte Behandlungsmethode, nämlich die Mercolintschürze, anempfiehlt, ja er hat sogar durch Beiersdorf \& Comp. eine besondere Form dieser Schürze erhalten, die er als die "Aachener Form" bezeichnet. Eine bessere Anerkennung der Richtigkeit meiner schon vor langer Zeit ausgesprochenen Ansicht, dass es das verdünstete, nicht das eingeriebene Quecksilber ist, welches vom Organismus absorbirt wird, kann ich nicht gern erhalten.

Gegenüber Jordan's Angabe, dass $\mathrm{Hg}$ erst nach einer 8-10tägigen Anwendung des Hg-Säckchens im Harn nachge- 
wiesen werden kann, will ich auf die grosse Anzahl Fälle hinweisen, wo ich schon nach einer 5-tägigen Anwendung des HgSäckchens eine recht erhebliche Menge $\mathrm{Hg}$ absorbirt gefunden habe, und ich glaube, dass ein jeder, der in der Ausführung von $\mathrm{Hg}$-Untersuchungen geübt ist, sich mittelst einer guten Methode davon überzeugen kann, dass sich schon bei einem 1-2-tägigen Tragen des $\mathrm{Hg}$-Säckchens in der Regel $\mathrm{Hg}$ im Harn nachweisen lässt, ganz wie bei einer wirklichen Einreibungscur, nota bene, wenn bei derselben eine so grosse Menge Salbe angewendet wird, dass ein Theil davon a ufgestrichen auf der Haut liegen bleibt. Jordan gibt an, dass bei der Anwendung der Einreibungscur Hg schon nach 4 Tagen, bei Anwendung von Sublimatinjectionen schon nach 2 Tagen im Harn nachgewiesen werden kann. Ich habe schon im Jahre 1886 gezeigt, dass bei der Einreibungscur $\mathrm{Hg}$ sich oft schon am Tage nach der ersten Einreibung im Harn nachweisen lässt, und dies ungeachtet ich damals eine so kleine Menge Salbe (3 Gr.) anwandte, dass nur ein verhältnissmässig geringer Theil davon aufgestrichen auf der Haut liegen bleiben konnte; ebenso habe ich damals dargethan, dass schon 2-3 Stunden nach den ersten Injectionen $\mathrm{Hg}$ im Harn nachzuweisen ist. - Wahrscheinlich sind verschiedene Untersuchungsmethoden und eine ungleiche Fertigkeit ${ }^{1}$ ) in der Ausführung dieser Untersuchungen die Ursache der Verschiedenheit des Ergebnisses von Jordan's und meinen Untersuchungen. Seine Angaben über die Absorption des Quecksilbers kann ich nicht als einen Beweis für eine langsam geschehende Absorption von $\mathrm{Hg}$ bei Anwendung von Hg-Säckchen anerkennen.

Die gegenwärtig wichtigste Streitfrage ist jedoch nicht die, ob Hg schnell und kräftig absorbirt wird oder nicht, sondern wie es bei der Einreibungscur, bei der Behandlung mit dem Säckchen, mit der Mercolintschürze u. s. w. in den Organismus gelangt.

Ich habe erst neulich in einem Aufsatz über die Mercolintschürze meine Stellung zu dieser Frage dargelegt, und ich will deshalb hier nur erwähnen, dass ich es anfangs ganz

1) Schillberg hat den chemischen Theil der in diesem Aufsatz erwähnten Harnuntersuchungen ausgeführt. 
unentschieden liess, ob dieses verdunstete Quecksilber durch die Lunge, durch die Haut oder durch beide absorbirt wird, später aber mebr und mehr der Ansicht zuneigte und mich davon überzeugt fühlte, dass diese Behandlungsformen hauptsächlich „Inhalationscuren“ sind. Auf Grund von Piccardi's Untersuchungen hebe ich jedocb in diesem Aufsatz die Wahrscheinlichkeit hervor, dass Hg-Dämpfe nicht nur durch die Respirationsorgane, sondern auch durch die Haut absorbirt werden; aber ich hebe auch hier, wie schon viele Male vorher hervor, dass dieses in praktischer Hinsicht eine verhältnissmässig geringe Bedeutung hat, da es für uns das Wichtigste ist, zu wissen, ob $\mathrm{Hg}$ wirklich in grosser Menge in den Organismus kommt, and da die $\mathrm{Hg}$-Säckchenmethode schon von Anfang an so eingerichtet gewesen ist, dass sie besser für die Haut- als für die Lungenabsorption passt, finde ich keine Veranlassung, diese meine Säckchenmethode abzuändern, trotzdem die Untersuchungen, die ich dieses Jahr ausgeführt habe, zeigen, dass Hg-Dämpfe durch die (intacte) Haut dringen können.

Ich will nun zu ein paar Anmerkungen übergehen, die Schuster in seinem letzten Aufsatz gegen mich erhoben hat. Er sagt hier: "Wenn man aber glaubt, dass das Tragen des Sackes (oder des Mercolints) während des. Herumgehens am Tage als Vortheil der Methode hinzugekommen, so begeht man m. E. einen Irrthum. Die Quecksilberdämpfe, welche hierbei aus dem Sacke oder Lint, also aus fast warmer Hauttemperatur in die umgebende Tagesluft gerathen, condensiren sich, wie schon Merget so sehr betont hat, zu Mercurtröpfchen, die eingeathmet nur mechanisch wirken, nicht aber den mit der eingeathmeten Luft in der Lunge vor sich gehenden Gaswechsel behufs Eindringens ins Blut mitmachen können. In Folge der beim Herumgehen in freier Luft vor sich gehenden Luftströmung kommen die auch in warmer Luft etwa entweichenden Hg-Gase wohl kaum zur Einathmung." Ich will hier nebenher bemerken, dass $\mathrm{Hg}$ schon bei einer Temperatur bedeutend unter dem Gefrierpunkt verdünstet und es durchaus nicht gesagt oder bewiesen ist, dass das $\mathrm{Hg}$, welches in der Tagesluft verdünstet, die ja oft, um nicht zu sagen zumeist, über 
dem Gefrierpunkt ist, in Mercurtropfen übergeht, die in den Mund und die Respirationsorgane kommen, ohne daselbst absorbirt werden zu können. Wenn dieses der Fall wäre, dürfte man ja im Speichel einer das Hg-Säckchen tragenden Person, wenn sie sich in Zimmerwärme befindet, nur $\mathrm{Hg}$, das durch die Speicheldrüsen eliminirt ist, aber wenn sie sich in kälterer äusserer Luft aufhält, ausser dieser eliminirten Hg-Menge auch diese Mercurtröpfchen, also eine viel grössere Menge $\mathrm{Hg}$ finden. Ich habe bis jetzt in dieser Hinsicht nur in einem Falle Untersuchungen angestellt. Ich sammelte von einem Patienten, der das Hg-Säckchen 25-30 Tage getragen hatte, in einen Becher Speichel, während er sich im warmen Krankenzimmer befand, und in einen anderen Becher Speichel, während er sich ein paar Tage in einer Luft ron nur ein paar Grad Wärme im Parke des Krankenhauses aufhielt. In beiden Proben zeigte sich eine minimale Menge $\mathrm{Hg}$ - so gross, wie ich sie stets im Speichel gefunden habe - und eine Erhöhung des $\mathrm{Hg}-$ Gehaltes durch Mercartröpfchen, die sich bei dem Aufenthalte des Patienten in der kalten Luft aus den Quecksilberdämpfen verdichtet hätten, liess sich in der betreffenden Probe nicht nachweisen. Ich führe das Ergebniss dieser Untersuchung hier an, ohne Gewicht darauf zu legen.

Aber Sch uster sagt: "Wenn man aber glaubt, dass das Tragen des Sackes (oder des Mercolints) während des Herumgehens am Tage als Vortheil der Methode hinzukomme, so begeht man m. E. einen Irrthum." Ich wende Hg-Säckchen im Winter z. B. nicht bei Grobarbeitern an, die sich des Winters oft bei beträchtlicher Kälte im Freien aufhalten müssen, wohl aber bringe ich sie in der kalten Jahreszeit bei Personen, die ihre Beschäftigung überwiegend innerhalb des Hauses haben, wie z. B. Kaufleuten, Beamten u. s. w., und bei den Patienten im Krankenhause zur Anwendung. Dass nun $\mathrm{Hg}$ von diesen Hg-Säckchen auch des Tages über absorbirt wird, habe ich gesagt, und es ist eine leichte Sache, sich davon zu überzeugen. Ich will hier die $\mathrm{Hg}$-Absorption in ein paar Fällen anführen. Zwei Frauen trugen $\mathrm{Hg}$-Säckchen nur des Tages, von $8 \mathrm{Uhr}$ des Morgens bis $8 \mathrm{Uhr}$ des Abends und nicht des Nachts in der Bettwärme. Beide waren in das 
Krankenhaus aufgenommen, wo sie des Tages über, gleich den iibrigen Patienten, mit verschiedenen Arbeiten an die Hand gehen mussten, sowohl innerhalb, wie ausserhalb des Krankenzimmers; die eine, die das Säckchen 35 Tage getragen, hatte ausserdem ein kleines hereditärsyphilitisches Kind zu pHlegen, so dass ihr wenig Zeit dazu übrig blieb, sich im Laufe des Tages zu legen und auszuruhen.

Fall I. A. E. wurde im Krankenhause St. Göran für einen Abscess behandelt, der einige Tage mit Sublimatlösung gespült wurde, woher es wohl kam, dass sie, noch ehe sie das Säckchen zu tragen begonnen, eine minimale Menge $\mathrm{Hg}$ im Harn hatte. Nach einem mit ihr getroffenen Uebereinkommen ${ }^{1}$ ) hat sie vom 24./IV. bis zum 9./V. täglich von $8 \mathrm{Uhr}$ Morgens bis um $8 \mathrm{Uhr}$ Abends ein 6 Gr. Ung. Hydr. (1 Hg., 2 Fett) enthaltendes Säckchen getragen. Den 23./IV. fanden sich in $380 \mathrm{Gr}$. Harn, 1.030 spec. Gew., einige sehr kleine Hg-Kügelchen. Den 29./IV. (nach 5-täg. Tragen des Säckchens) fanden sich in $320 \mathrm{Gr}$. Harn, 1.015 spec. Gew., recht viele kleine Hg-Kügelchen. 3./V. (nach 15-täg. Tragen des Säckchens) fanden sich in 320 Gr. Harn, 1.028 spec. Gew.; eine grosse Menge Hg-Kügelchen, darunter mehrere grosse. Also eine recht kräftige Absorption von $\mathrm{Hg}$.

Fall II. G. E. wurde wegen Roseola und Pap. mucosae in das Krankenhaus aufgenommen und trug das Säckchen vom 24./IV. bis zum 29./V. von $8 \mathrm{Uhr}$ Morgens bis um $8 \mathrm{Uhr}$ Abends. Den 23./IV. in $330 \mathrm{Gr}$. Harn, 1.018 spec. Gew., einige Kügelchea. Den 29./IV. in 315 Gr. Harn, 1.023 spec. Gew., recbt viele Kügelchen, einige recht grosse. Den 9./V. in 310 Gr. Harn, 1.026 spec. Gew., eine grosse Menge Kügelchen, zwei grosse. Den 14./V. in 380 Gr. Harn, 1.019 spec. Gew., eine bedeutende Menge recht grosse Hg Kügelchen. Den 19./V. in $365 \mathrm{Gr}$. Harn, 1.016 spec. Gew., ein sehr grosses and eine bedeutende Menge kleinere Hg-Kügelchen. Den 24./V. in $360 \mathrm{Gr}$. Harn, 1.017 spec. Gew., eine höchst bedeutende Menge Hg. Kügelehen, darunter viele recht grosse. Den 29. V. in $375 \mathrm{Gr}$. Harn, 1.013 spec. Gew., eine bedeutende Menge Kügelchen, darunter viele recht grosse.

Wir finden also in beiden Fällen eine ziemlich kräftige Absorption von $\mathrm{Hg}$, ungeachtet das Säckchen nur des

1) Da in einer ausländischen Zeitschrift Vorwürfe gegen mich erhoben worden sind, weil ich den einen oder anderen Versuch angestellt, dessen Zweck wissenschaftliche Forschung, nicht therapeutischer Nutzen für den Patienten war, will ich hier erklären, dass ich nie solehe Versuche ausgeführt habe oder ausführe, ohne die Einwilligung des Patienten dazu erhalten zu haben und ohne ihn (oder sie) dafür zn bezahlen (siehe hierüber meinen Aufsatz vom Jahre 1884: Einige Untersuchungen über die pathogenen Microben der Gonorrhoe). Nord. Med. Arkiv. 
Tages ïber getragen worden ist. Unter solchen Verhältnissen zu sagen: "Wenn man aber glaubt, dass das Tragen des Sackes (oder des Mercolints) während des Herumgehens am Tage als ein Vortheil der Methode hinzukomme, so begeht man m. E. einen Irrthum," ist nicht berechtigt, im Gegentheil, es liegt klar zu Tage, dass man, wenn man bei dieser Methode eine richtig kräftige $\mathrm{Hg}$-Absorption haben will, das Säckchen von dem Patienten nicht nur des Nachts in der Bettwärme, sondern auch, in den Fällen, wo dieses geschehen kann, des Tages über tragen lassen muss.

Die Frage ist nun die: In welcher Weise, auf welchem Wege gelangt das $\mathrm{Hg}$ bei Anwendung der Säckchenmethode, vor allem aber der Einreibungscur, in den Organismus? Um diese Frage zu beantworten, hat Schuster ein Experiment gemacht, aus welchem er folgenden Schlnss zieht: "Hierdurch ist die Fähigkeit der Haut, Quecksilber zu absorbiren, erwiesen; demnach fällt die Hauptstütze der Quecksilbereinathmungscur mittels Quecksilbersäckchen oder Lappen gegenüber der Einreibecur."

Ich verstehe nicht recht, was Schuster mit diesem Experiment eigentlich beweisen will. Es ist ja wohl etwas eigenthümlich, dass er zu diesem seinen Versuch nicht die wirkliche Einreibungscur, sondern Herxheimer's Modification derselben (die Klatsch-Methode) anwendet, doch will ich hierauf weiter kein Gewicht legen. Aber beweist nun dieses Experiment nach Schuster's Ansicht, dass es das eingeriebene (resp. eingeklatschte) Quecksilber ist, welches absorbirt wird, oder soll es beweisen, dass die entwickelten $\mathrm{Hg}$-Dämpfe durch die Haut absorbirt werden? Schuster drückt sich hier recht dunkel aus, so dass man den Schluss, den er zieht, in beiden Richtungen deuten kann.

Schuster hat indessen sein Experiment in folgender Weise ausgeführt: "Abends vor Schlafengehen wurde ein Oberschenkel mit Wasser befeuchtet, dann Mercurseifensalbe mittels eines Holzspatels rasch aufgestrichen; die aufgestrichene Stelle, resp. der ganze Oberschenkel mit dichtem weichem Papier umgeben, darüber kam eine Einpackung mit Kautschukpapier; nun wurde auf den so gesicherten Oberschenkel mittels 
eines breiten, langen Holzspatels fünf Minuten lang geklopft, resp. geklatscht, dann mittels dreier Gazebinden die Linpackung so umwickelt, dass eine Verrutschung ausgeschlossen war. Das geschah im Sommer spät Abends in einem an das Schlafzimmer anstossenden Zimmer. Morgens Früh wurde wieder in dem anstossenden Zimmer der Verband abgenommen, die auf der Haut lagernde Salbe mittels in Benzin getauchter Watte so lange abgewaschen, bis die Watte weiss blieb. Diese Weise wurde 9 mal mit 5 und 7.5 Gr. betragender Seifensalbe wiederholt. Der darauf zur Untersuchung auf $\mathrm{Hg}$ weggesandte Harn ergab einen breiten, schönen Jod-Hg-Ring.“

Ich habe dieses Experiment nachgemacht, in ganz derselben Weise, wie es ron Schuster ausgeführt worden ist, doch habe ich anstatt Mercurseifensalbe, die mir nicht zur Verfügung stand, Ung. Hydr. (1:2) angewandt. In der Regel wurde die Procedur um 8 Uhr Abends ausgeführt, die Bandage am Vormittag darauf zwischen 9 und $10 \mathrm{Uhr}$ abgenommen und dann die Haut abgewaschen.

Fall III. Den 18.|IV. um 2 Uhr Nachm. wurde die erste Aufklatschung von Hg-Salbe gemacht. Den 23./IV. fanden sich in $350 \mathrm{Gr}$. Harn, spec. Gew. 1.020, recht viele $\mathrm{Hg}$-Kügelchen, darunter einige recht grosse. Den 28./IV. fanden sich in 335 Gr. Harn, spec. Gew. 1.029, eine grosse Menge meist kleine Hg-Kügelchen. In Folge des Waschens des Beines mit Benzin war am linken Oberschenkel eine bedentende Reizung mit einer Menge Hautlosigkeiten entstanden, weshalb an den folgenden 5 Tagen der Process an dem rechten Oberschenkel ausgeführt wurde. Den 3./V. fanden sich in $350 \mathrm{Gr}$. Harn, spec. Gew. 1.020, eine Menge Hg Kügelchen, darunter viele recht grosse. Nun wurde, mit der Einklatschung aufgehört, da sich auch an diesem Schenkel eine recht bedeutende Hautreizung zeigte. Den 5./V. verliess der Patient das Krankenhaus; in $300 \mathrm{Gr}$. Harn, spec. Gew. 1.015, fanden sich an diesem Tage eine grosse Menge Hg-Kügelchen, darunter viele recht grosse.

Ich kann sagen, dass ich überrascht war, hier eine so kräftige Absorption ron $\mathrm{Hg}$ zu finden. Aber nun entstand die Frage: auf welchem Wege hat dieses $\mathrm{Hg}$ in den Organismus gelangen können? Es lässt sich denken, dass das eingeklatschte (resp. eingeriebene) Quecksilber durch die Haut absorbirt worden ist, aber es lässt sich auch denken, dass sich unter diesem Verbande mit Kautschukpapier eine Menge Hg-Dämpfe entwickelt haben, die möglicherweise durch die Haut des Oberschenkels absorbirt worden sind, und ebenso lässt es sich 
denken, dass die aufgelegte Bandage nicht hinreichend dicht geschlossen hat und es dadurch einem Theil der Hg-Dämpfe möglich geworden ist, sich trotz derselben, zwischen ihn hindurch und an ihren Seiten, vorbei, zu verbreiten, so dass sie dann durch die Respirationsorgane und vielleicht auch die übrige Haut absorbirt werden konnten. Um auf diese Frage möglicherweise eine Antwort geben zu können, habe ich folgende Versuche angestellt.

Sollte es das in die Haut eingeriebene Quecksilber sein, das absorbirt wird, so müsste diese Absorption auch geschehen können, wenn das nach der Einklatschung (resp. Einreibung) auf der Haut lagernde Quecksilber sorgfältig abgewaschen wird. - Um zu erfahren, wie es sich hiermit verhält (oder richtiger, um dieses zu controliren, denn ich hatte voraus Versuche in dieser Richtung ausgeführt), liess ich bei einer Frau vom 17./V. bis zum 1./VI. täglich 6 Gr. Ung. Hydr. $(1: 2)$ in ganz derselben Weise wie in Fall III auf dem einen Schenkel einklatschen, nach der Einklatschung aber das Kautschukpapier wegnehmen und die auf der. Haut lagernde Salbe sorgfältig abwaschen, doch nicht mit Benzin, um die Haut nicht zu reizen, sondern mit Seife und Wasser. - Wäre es nun das eingeklatschte (resp. eingeriebene) Quecksilber, welches absorbirt wird, so müsste sich ja auch in diesem Falle eine mehr oder weniger kräftige Hg-Absorption zeigen. Das Ergebnis dieses Versuches gestaltete sich folgendermassen:

Fall IV. V. P., war Monate hindurch im Krankenhause St. Göran wegen Gonorrhöe in der Urethra, im Cervikal-Canale u. s. w. sowie wegen einer schweren, mit Endocarditis verbundenen gonorrhoischen Allgemeininfection behandelt worden, wobei sie Wochen hindurch täglich bedeutende Temperaturwechselungen gehabt hatte. Zeitweise hatten in demselben Zimmer wie sie Patienten gelegen, welche die Hg-Behandlung durchmachten. Dieses war sicher die Ursache, dass sie beim Beginn des Versuches eine minimale Menge $\mathrm{Hg}$ im Harn hatte. Es fanden sich beim Beginn des Versuches den 17./V. in $200 \mathrm{Gr}$. Harn, spec. Gew. 1.026, einige sehr kleine Hg-Kügelchen; an diesem Tage wurde mit dem Einklatschen begonnen. Den 22./V. waren in 365 Gr. Harn, spec. Gew. 1.015, ein paar kleine Hg-Kügelchen zu entdecken. Den 27.|V. liessen sich in $370 \mathrm{Gr}$. Harn, spec. Gew. 1.025, keine Hg-Kügelchen entdecken. ')

1) Tch will hier bemerken, dass man, wenn die Hg-Menge so minimal wie in diesem Falle ist, unmöglich sagen kann, dass sich kein $\mathrm{Hg}$ im 
Den 1./VI. fanden sich in $360 \mathrm{Gr}$. Harn, resp. Gew. 1•015, einige minimale Kügelchen. Die Patientin wurde an diesem Tage aus dem Krankenhause entlassen.

Also war bei diesem Versuch keine merkbare Absorption ron $\mathrm{Hg}$ nachzuweisen, was wohl deutlich darthut, dass das eingeklatschte resp. eingeriebene Quecksilber nicht absorbirt wird.

Man muss also wohl annehmen, dass die Absorption von $\mathrm{Hg}$ in Fall III eine Absorption von entwickelten Hg-Dämpfen gewesen ist. Hierfür fand sich ja auch eine grosse Möglichkeit, denn unter einem gut angelegten Kautschukrerband ist die Temperatur wahrscheinlich sehr hoch. Ich habe diese Temperatur dreimal gemessen, indem ich bei verschiedenen Personen ein Maximithermometer nächst der Haut unter die Bandage legen liess, welches die Nacht über liegen blieb; es zeigte sich hierbei, dass die Maximitemperatur unter der Bandage in einem Falle (III) $36^{\circ} \mathrm{C}$, in dem zweiten (V) $35^{\cdot} 7^{\circ} \mathrm{C}$. und in dem dritten (VI) $35^{\circ} 6^{\circ} \mathrm{C}$. gewesen war. Dass bei einer so hohen Temperatur eine grosse Menge Hg-Dämpfe gebildet werden, unterliegt ja keinem Zweifel; aber nun war die Frage die: bleiben diese Hg-Dämpfe in überwiegender Menge unter der Bandage, oder verbreiten sie sich ausserhalb derselben, so dass sie den Körper unter der Bettdecke überall umgeben und also auch an anderen Stellen als der mitKautschuk überdeckten Hautpartie absorbirt werden können?

Um dieses zu ermitteln, ging ich in folgender Weise zuwege; ich nahm kleine Schachteln mit doppelten Böden und bohrte in die Wände zwischen den Böden eine Menge kleine Löcher, damit die Hg-Dämpfe unbehindert durch sie passiren konnten, und ebenso bohrte ich solche Löcher für diesen Zweck in den oberen Boden. Ich konnte hierdurch sicher sein, dass zwischen den oberen Boden und den ordentlich aufgebundenen Deckel der Schachtel nur Hg-Dämpfe kommen konnten, selbst wenn etwas Salbe zwischen die beiden Böden kommen sollte. Zwischen den oberen Boden und den Deckel wurden nun zwei kleine, 2-3 Ctm. lange feine Kupferdrähte

Harn findet, ungeachtet man trotz der sorgfältigsten Untersuchung auch nicht das geringste Kügelchen zu entdecken vermag. 
gelegt, die unmittelbar vorher in warmer Salzsäurelösung rein gemacht worden waren (es war controlirt worden, dass sich an ihnen kein $\mathrm{Hg}$ fand). Eine solche Schachtel wurde auf der Haut unter der Kautschukbandage festgebunden und eine andere an einer um den Hals gebundenen Schnur befestigt; beide Schachteln blieben die Nacht über an ihren Plätzen. Am folgenden Tage wurden sie weggenommen und die feinen Kupferdrähte, die in ihnen gelegen, in je eine feine Glasröhre gebracht, in derselben erhitzt und dann mikroskopisch untersucht, um zu sehen, ob sich an ihnen abgesetztes Hg fand. Bei wiederholt ausgeführten Versuchen zeigte es sich, dass sich an die Kupferdrähte der unter der Bandage festgebundenen Schachtel viel Quecksilber abgesetzt hatte, während sich an den Kupferdrähten der an dem Halse getragenen Schachtel nur eine minimale Menge Quecksilber fand. Als Beispiel will ich hier von Fall III anführen, dass den 2./V. 2 Uhr 30 Min. Nachm. der Kautschukverband mit einer unter ihm auf der Haut liegenden Schachtel mit Kupferdrähten eingelegt und eine solche Schachtel um den Hals gebunden wurde. Den $3 . / \mathrm{V}$. $8 \mathrm{Uhr}$ Vorm. wurden die Schachteln weggenommen und zeigten sich dann die Kupferdrähte der auf dem Oberschenkel placirt gewesenen nicht blank, sondern etwas grauweiss, und ron ihnen sublimirten bei Erhitzung in einer Glasröhre eine höchst bedeutende Menge $\mathrm{Hg}$-Kügelchen, doch im allgemeinen kleinere. Die Kupferdrähte in der am Halse getragenen Schachtel waren makroskopisch unverändert, und an ihnen liessen sich bei der Sublimirung in einer Glasröhre nur ein paar kleine Hg-Kügelchen entdecken.

Unter solchen Umständen liess sich nicht gern eine eigentliche Absorption von Hg-Dämpfen durch die Respirationsorgane (oder die übrige Haut) annehmen, sondern es muss die Absorption durch die Haut des mit Kautschuk umwickelten Oberschenkels geschehen sein.

War dieses nun wirklich der Fall, so müsste natürlicherweise eine Absorption, ja! eine gleich kräftige Absorption stattfinden, wenn ein kleines Säckchen mit Hg-Salbe oder Mercuriol unter einem Kautschukverband auf den Oberschenkel gelegt würde. Um dieses zu ermitteln, führte ich folgenden Versuch aus. 
F all V. S. M., wurde in das Krankenhaus St. Göran wegen Clcus molle aufgenommen. Den 16./V. wurde auf den einen Oberschenkel unmittelbar auf die Haut ein Theil Gaze gelegt und darauf das Hg-Säckchen mit 6 Gr. Ung. Hydr. placirt, damit von demselben keine Salbe direct mit der Haut in Berührung komme; über das Säckchen wurde Wachstaffet gebreitet und das Ganze dann wie in Fall III mit Gazebinden überbunden. Die Hg-Bandage wurde in der Regel von $8 \mathrm{Uhr}$ Nachm. bis um $8 \mathrm{Uhr}$ Vorm. getragen. Den 16./V. fanden sich in $330 \mathrm{Gr}$. Harn, spec. Gew. 1.024, keine $\mathrm{Hg}-K u ̈ g e l c h e n . ~ D e n ~ 21 . / V$. fanden sich in $365 \mathrm{Gr}$. Harn, spec. Gew. 1*019, eine grosse Menge, zum Theil kleine Hg-Kügelchen. Ziemlich grosse Reizung in der Haut des Oberschenkels, weshalb das Säckchen anf den andern Oberschenkel gelegt wird. Den 31./V. fanden sich in 360 Gr. Harn, spee. Gew. 1.016, eine Menge Hg-Kügelchen, darunter viele recht grosse. Höre mit dem Auflegen des Säckchens auf. Den 3./VI. fanden sich in $360 \mathrm{Gr}$. Harn, spec. Gew. 1.022, eine grosse Menge Kügelchen, darunter mehrere recht grosse.

Also auch in diesem Falle eine ziemlich kräftige Absorption; dass die Hg-Dämpfe auch hier grösstentheils unter dem Verbande blieben, zeigten Versuche mit den erwähnten Schachteln. So trug die Patientin vom 17./N. von $8 \mathrm{Uhr}$ Nachm. bis zum 18./V. 10 Uhr 30 Min. Vorm. Schachteln unter der Bandage, wobei sich an den Kupferdrähten eine sebr bedeutende Menge Hg-Kügelchen, worunter sich einige recht grosse befanden, nachweisen liessen, während bei anderen Versuchen, z. B. rom 23./V. bis zum 24./V., wo die Schachtel auf der Brust getragen wurde, nur ein einziges kleines $\mathrm{Hg}$-Kügelchen entdeckt werden konnte; dieses war auch der Fall bei einem Versuch vom 25./V. bis zum 26./V. Hier ist es ja augenscheinlich, dass Hg-Dämpfe durch die Haut des Schenkels absorbirt worden sind. Ebenso augenscheinlich war dieses bei einem entsprechenden Versuch mit einem mit 5 Gr. Mercuriol täglich gefülten Säckchen, das vom $16 . / \mathrm{V}$. bis zum $31 . / \mathrm{V}$. getragen wurde.

Fall VI. T. E. Den 16./V. in 370 Gr. Harn, spec. Gew. 1.017, keine Hg-Kügelchen. Den 21./V. in $360 \mathrm{Gr}$. Harn, spec. Gew. 1•016, eine grosse Menge Kügelchen, einige nicht klein. Den $31 . / \mathrm{V}$. in $365 \mathrm{Gr}$. Harn, spec. Gew. 1.027, eine grosse Menge Kügelchen, einige recht gross. Es wird mit dem Tragen des Säckchens aufgehört: Den 3./VI. in 365 Gr. Harn, spec. Gew. 1.029, eine grosse Menge $\mathrm{Hg}-\mathrm{Kühgelchen,} \mathrm{darunter} \mathrm{einige}$ ziemlich grosse.

Wir finden also eine ziemtich kräftige $\mathrm{Hg}$-Absorption. Entsprechende Proben mit Schachteln wurden auch hier mit 
dem Resultat gemacht, dass sich an den Kupferdrähten der auf dem Schenkel getragenen Schachtel viel $\mathrm{Hg}$ fand, während sich z. B. den 23./V. an den Kupferdrähten der auf der Brust getragenen Schachtel nur ein einziges Kügelchen nachweisen liess. Aber den 25./V. zeigten sich an den Kupferdrähten der auf der Brust getragenen Schachtel eine Menge kleine HgKügelchen; die Erklärung war nicht schwer; wie nämlich die Patientin angab, hatte sich die Bandage im Laufe der Nacht verrïckt, und dabei waren natürlicherweise die Hg.Dämpfe unter der Bandage hervorgeströmt, unter der Bettdecke uiberall hin gedrungen und in dieser Weise mit $d_{3 n}$ Kupferdrähten in der Schachtel auf der Brust in Berihrung gekommen.

Um grössere Gewissheit hierïber zu erhalten, führte ich schliesslich rom 24./V. bis zum 8./VI. einen Versuch aus, was in ganz derselben Weise wie in Fall III geschah, obschon die Salbe jetzt nicht mit Benzin, sondern mit Seifenwasser abgewaschen wurde.

F all VII. H. T. Den 25./V. in 340 Gr. Harn, spec. Gew. 1.017, keine Hg-Kügelchen. Den 30./V. in $370 \mathrm{Gr}$. Harn, spec. Gew. 1.017, eine Menge Hg-Kügelchen, die meisten klein. Den 3./VI. in 360 Gr. Harn, spec. Gew. 1.017, eine grosse Menge Kügelchen, mehrere recht gross. Den 8./VI. in 360 Gr. Harn, spec. Gew. 1.013, eine grosse Menge Kügelchen, ein paar gross, mehrere recht gross.

Also war das Ergebniss dieses Versuches vollständig dem der früheren gleich. Die Untersuchung mit den Schachteln gab dasselbe Resultat wie in den vorhergehenden Fällen.

Es lässt sich wohl nicht gern bezweifeln, dass $\mathrm{Hg}$ durch die Haut absorbirt worden ist, doch nur verdünstetes, nicht eingeriebenes oder eingeklatschtes.

Man as se in hat schon hervorgehoben, dass beiSchu ster's Versuchen die Haut nicht intact gewesen sein kann; in meinen Fällen III und V war die Haut sehr gereizt, theilweise excoriirt, und dieses hat wohl zur Hg-Absorption in diesen Fällen beigetragen; in den Fällen VI und VII dagegen zeigte sich die Haut dem blossen Auge intact, und trotzdem fand hier eine kräftige Absorption statt; hervorzuheben sind jedoch auch in diesen Fällen die Ausnahmeverhältnisse (der Kautschukverband), unter welchen die Absorption geschehen ist. 
Hat Schuster durch seinen Versuch darthun wöllen, dass es das eingeklatschte, resp. eingeriebene Quecksilber ist, welches absorbirt wird, so kann ich nicht in den Schluss einstimmen, den è in diesem Falle aus seinem Versuche gezogen hat; will er mit seinem Versuch hingegen beweisen, dass es die unter der Bandage gebildeten Hg-Dämpfe sind, die absorbirt werden, so bin ich vollkommen mit ihm einig. Doch will ich dann hervorheben, dass uns diese Versuche einen ziemlich deutlichen Wink geben, unsere Patienten nicht länger mit diesen unbehaglichen Einreibungen zu plagen, sondern uns damit zu begnügen, sie Hg-Dämpfen auszusetzen.

Zeigt es sich nun, dass $\mathrm{Hg}$-Dämpfe durch die (wenigstens dem Aussehen nach intacte) Haut des Oberschenkels absorbirt werden können, so ist es ja klar, dass ihre Absorption auch durch die Haut an anderen Stellen des Körpers geschehen kann. Bei der Anwendung der Hg-Säckchenmethode findet sich solchergestalt für das Quecksilber die Möglichkeit, von grossen Hautflächen absorbirt zu werden, denn die Hg-Dämpfe bleiben nicht unter dem $\mathrm{Hg}$-Säckchen, sondern sie verbreiten sich, wie mehrere Versuche mit meinen Schachteln gezeigt haben, über alle Theile des Körpers. Ich will hier von diesen Versuchen nur ein paar anführen.

E. S. hatte in der Nacht vom 29./V. zum 30.|V. ein Hg-Säckchen auf dem Rücken und eine von meinen Schachteln, um den Hals gebunden, auf der Brust; an den. Kupferdrähten dieser Schachtel fand sich am Morgen eine bedeutende Menge kleiner Hg-Kügelchen. In der Nacht vom 30./V. zum 31./V. hatte der Patient das Säckchen auf der Brust und die Schachtel zwischen den Schenkeln; am Morgen zeigte sich an den Kupferdrähten eine bedeutende Menge kleinerer $\mathbf{H g}$-Kügelchen.

A. S. hatte in der Nacht vom 29./V. zum 30/V. ein Hg-Säckchen auf der Brust und eine Schachtel zwischen den Oberschenkeln; am Morgen fanden sich an den Kupferdrähten der Schachtel eine grosse Menge kleinere Hg-Kügelchen. In der Nacht vom 30./V. zum 31/N hatte dér Patient das $\mathrm{Hg}$ Säckchen auf dem Rücken und die Schachtel mit den Kupferdrähten auf der. Brust; am Morgen zeigten sich an den Drähten eine sehr bedeutende Menge sehr kleineHg-Kügelchen.

Man kann ja fragen: kann die Auffassung richtig sein, dass an den Stellen, wo das Hg-Säckchen liegt, eine grössere Absorption von $\mathrm{Hg}$ stattfindet, für welche Auffassung man eine Stïtze in dem Umstande hat finden wollen, dass die Symptome 
an diesen Stellen eher als an anderen Körpertheilen verschwinden? Ich habe zwar in Betreff dieser Frage meine Ansicht über das verschiedene Verschwinden der syphilitischen Symptome in meinem Aufsatz über die Mercolintschurze ausgesprochen, eine Ansicht, welche ich in der Hauptsache festhalte, doch gebe ich bereitwillig zu, dass, wenn $\mathrm{Hg}$-Dämpfe unter dem Säckchen in grösserer Menge als an anderen Stellen des Körpers nachgewiesen werden können. von der Haut unter dem Säckchen $\mathrm{Hg}$ auch ganz wahrscheinlich in grösserer Menge als von der Haut an anderen Theilen des Körpers absorbirt wird. Um dieses zu ermitteln habe ich einen Theil Versuche mit meinen Schachteln mit Kupferdraht ausgeführt. Zwar besitzt diese Untersuchungsmethode keinen wissenschaftlichen Werth, doch kann sie uns gleichwohl eine approximative Erklärung darüber geben, wie es sich hiermit verbält. Wird an den Kupferdrähten in den Schachteln auf den verschiedenen Körpertheilen beinahe constant ein verschiedener $\mathrm{Hg}$-Ansatz gefunden, so dürfte dieses ein ziemlich starker Wahrscheinlichkeitsbeweis dafür sein, dass sich auf den verschiedenen Theilen des Körpers eine verschieden grosse Menge $\mathrm{Hg}$-Dämpfe findet und also auf ihnen wahrscheinlich auch eine verschieden grosse Absorption von Hg-Dämpfen stattfindet.

Ich habe nun in einer Menge von Fällen bei denselben Patienten eine Schachtel mit Kupferdrähten unter dem HgSäckchen auf der Brust und eine andere zwischen den Oberschenkeln placirt. Auch wenn es nun ein paarmal vorgekommen ist, dass sich in dem Hg-Ansatz an den Kupferdrähten in der auf der Brust und in der zwischen den Schenkeln und an anderen Stellen placirten Schachtel kein augenscheinlicher Unterschied fand, so ist dieses doch meistens der Fall gewesen. Es hat sich nämlich gezeigt, dass von den Kupferdrähten in der Schachtel auf der Brust eine bedeutende Menge Hg-Kügelchen sublimirten, während die Anzahl der von den Drähten in der Schachtel zwischen den Oberschenkeln sublimirten Kügelchen ersichtlich geringer war. Diese Versuche lassen es also als höchst wahrscbeinlich erscheinen, dass mehr Hg-Dämpfe unter dem Hg-Säckchen absorbirt werden und dass dieses zu einem schnelleren Verschwinden der Symptome 
auf der Brust und dem Rücken bei der Behandlung mit HgSäckchen beitragen kann.

Aber vielleicht fühlt man sich versucht, noch eine Frage aufzuwerfen, nämlich die: Geschieht alle Hg-Absorption bei der Behandlung mit diesen Hg-Säckchen durch die Haut, und hat vielleicht Spiegler Recht, wenn er sagt, "dass diese Methode vielleicht die reinste endermatische Methode ist!" Sie würde je dann durchaus keine Inhalationscur sein. Allzuviele Beweise finden sich, dass die $\mathrm{Hg}$-Dämpfe durch die Respirationsorgane in den Körper gelangen, um diesen Weg für die Hg-Absorption unterschätzen zu können. Gegenwärtig ist es indessen unmöglich zu entscheiden, wie viel $\mathrm{Hg}$ auf dem einen, und wie viel auf dem anderen Wege absorbirt wird. Glücklicherweise ist dieses für uns in praktischer Hinsicht nicht von grosser Bedeutung, denn das Wichtigste ist es ja, wie ich oben gesagt habe, zu wissen, dass $\mathrm{Hg}$ bei der Anwendung dieser Methode, die von vornherein so eingerichtet ist, dass sie sich sowohl für eine Absorption der $\mathrm{Hg}$-Dämpfe, durch die Haut, wie durch die Respirationsorgane eignet, schnell und kräftig absorbirt wird.

Man könnte ja sagen, dass es sich empfehle, Hg-Säckchen auch auf anderen Körpertheilen, z. B. den Extremitäten, wenn sich dort schwerere Symptome finden, anzuwenden. Dieses ist wohl möglich, doch ist es, da wir solche Mittel wie Un n a's Carbol-Hg-Pflastermulle haben, die wir in Fällen, wo eine locale Behandlung erforderlich ist, anwenden können, weniger praktisch. In welcher $\mathrm{W}$ eise das $\mathrm{Hg}$ bei der Anwendung dieses Mittels absorbirt wird, wissen wir nicht, doch ist es wohl wahrscheinlich, dass sich unter dem Guttaperchazeug $\mathrm{Hg}$ Dämpfe entwickeln und dann durch die Haut in den Organismus gelangen, was um so leichter gescbehen kann, als die Epidermisschicht unter der Pflastermulle oft mehr oder weniger macerirt wird.

Auch wenn nun diese meine Untersuchungen ebensowohl wie die Untersuchungen anderer Forscher für die Absorption des Quecksilbers eine andere Erklärung als die geben, die ich bisher für richtig angesehen habe, so geben sie mir doch keine Veranlassung, die Hg-Säckchenmethode zu verändern, 
und ebensowenig geben sie Anlass zu einer Veränderung der Anwendung der Mercolintschürze, so wie dieselbe in meinem oben erwähnten Aufsatz von mir vorgeschlagen worden ist. Weit entfernt davon, die Berechtigung der Anwendung dieser auf die Verdünstung des $\mathrm{Hg}$ basirten Behandlungsmethoden zu verringern, scheinen mir diese Untersuchungen die Berechtigung ihrer Anwendung zu erhöhen.

Man hat aber auch andere Anmerkungen gegen meine Hg-Säckchenmethode erhoben. Man hat nämlich gesagt, dass $\mathrm{Hg}$ bei Anwendung dieser Methode zwar absorbirt, aber bald wieder eliminirt wird, daher eine Wirkung, die eine längere Zeit bestehen bleibt, von einer solchen Behandlung nicht zu erwarten ist.

Jordan hat in dieser Hinsicht eine Angabe gemacht, die ich zwar nicht erst zu widerlegen brauche, auf die ich hier aber hinweisen will. Er sucht hervorzuheben, dass sich in der Remanenz des $\mathrm{Hg}$ bei der Behandlung mit Säckchen und mit Einreibungen ein Unterschied findet und dass dieser Unterschied zu Gunsten für die Einreibungscur spricht. Er sagt nämlich l. c. pag. 363: "W elander gibt an, dass nach Injectionen, insbesondere nach solchen mit unlöslichen Präparaten, das Quecksilber am längsten, nämlich zwei Monate, im Körper remavire, dass aber auch nach seinen Salbensäckchen das Quecksilber in den ersten. Wochen in ganz grosser Menge remanire. Nach Lanz remanirt das $\mathrm{Hg}$ nach Einreibungen noch viel länger; so konnte er in einem Falle nach freilich mehrfachen Hg-Curen, theils Spritz-, theils Einreibungscuren, von denen aber die letzte eine Einreibungscur gewesen war, ein halbes Jahr nach Abschluss derselben noch Spuren von Hg im Urin finden." Es geht ja hieraus hervor, dass Jordan die Bedeutung des Unterschiedes zwischen einer grossen Menge und nur Spuren eliminirten (d. h. remanirenden) Hg nicht richtig auffasst; ebenso scheint es, als ob er meine Angabe, dass "in den ersten Wochen $\mathrm{Hg}$ in ganz grosser Menge im Körper remanirt", dahin deutet, als ob hier meiner Ansicht nach alle $\mathrm{Hg}$-Elimination abgeschlossen sei. Niemals habe ich 
gesagt, dass nach diesen Injectionen von unlöslichen Salzen "Quecksilber am längsten, nämlich zwei Monate, im Körper remanire"; im Gegentheil, ich habe stets hervorgehoben, dass das $\mathrm{Hg}$ immer lange, mehrere Monate, in einer sich mehr und mehr vermindernden Menge im Körper remanirt.

Da indessen meine Untersuchungen vom Jahre 1885 so wenig bekannt sind, will ich hier über die Remanenz, Absorption und Elimination des Quecksilbers einige Worte aus meinem Aufsatz in Nord. Med. Arkiv, 1886, anführen, in welchem Aufsatze ich zeige, dass das Quecksilber, unabhängig von der angewendeten Behandlungsform, stets constant eliminirt wird. Zu dieser Zeit war allgemein die Ansicht herrschend, dass das $\mathrm{Hg}$ sehr unregelmässig eliminirt werde und dass es 13--15 Jahre lang im Organismus remaniren könne.

Erst will ich jedoch Einspruch gegen einige Worte von Gschwend einlegen. Derselbe sagt nämlich anlässlich einer Aeusserung von mir über die constante Elimination des Quecksilbers: „Allein Welanders Argument beruht auf einer irrthümlichen Voraussetzung. Schuster stellt nämlich damals fest, dass das Quecksilber bis zu sechs, ja sogar bis zu acht Monaten nach der Einreibecur constant eliminirt wird. Welander aber glaubte, dass diese Quecksilberelimination nach jeglicher Art von Quecksilberbehandlung stattfinde und somit auch auf die Behandlung mittelst der Welander'schen Säckchen Bezug haben müsse, während $S \mathrm{chuster}$ doch nur von Einreibungen spricht." Ich will in Bezug hierauf nur erklären, dass ich nicht bloss geglaubt, sondern auch bewiesen habe, dass nach Anwendung aller der verschiedenen $\mathrm{Hg}$ Methoden; die ich geprüft, $\mathrm{Hg}$ constant eliminirt. wird, so z. B. nach der Anwendung verschiedener Formen von $\mathrm{Hg}$-Pillen, mehrerer Arten von Injectionen löslicher und unlöslicher $\mathrm{Hg}$ Salze, Einreibung und Ueberstreichung mit Hg-Salbe, HgSäckchen, Mercolintschürze u. s. w. Ich bitte, hier auf meinen ersten Aufsatz hierüber im Jahre 1886 sowie auf einige andere, in den folgenden Jahren veröffentlichte Aufsätze und die Angaben hinweisen zu dürfen, die ich hier weiter hinten über die Hg-Säckchenmethode mittheile. Ich habe meine Aeusserung weder auf eine irrthümliche, noch auf eine nicht irrthümliche 
Voraussetzung, sondern auf von mir ausgeführte Untersuchungen gestiitzt.

Hinsichtlich der Remanenz (resp. Elimination) des Quecksilbers äusserte ich in dem genannten Aufsatz im Jahre 1886, nachdem ich ïber die Angabe berichtet, dass Hg 13-15 Jahre im Organismus remaniren könne : „Es lässt sich nicht gerne denken, dass diese Angaben richtig sein können und dass die Autoren die Angaben der Patienten über die Zeit, wo sie wirklich unter der einen oder anderen Form $\mathrm{Hg}$ das letzte Mal erhalten, genau controlirt haben."

"Es ist natürlicherweise von dem grössten Gewicht, sich, wenn man beurtheilen will, wie lange $\mathrm{Hg}$ im Körper remaniren kann, hierüber äussert genan zu unterrichten. Es ist durchaus nicht hinreichend, von den Patienten oder aus einem Journal zu erfahren, dass vor so und so viel Monaten oder Jahren so und so viel $\mathrm{Hg}$ in der oder der Form gegeben wurde, d. h. dass der Patient damals seine letzte $\mathrm{Hg}$-Behandlung durchmachte. Man würde da leicht eineu grossen Irrthum begehen können, und als Beispiel will ich hier Fall 28 nennen, wo die Patientin im Jahre 1869 eine Schmiercur durchmachte und Jodurpillen bekam, worauf sie nicht mehr wegen Syphilis mit $\mathrm{Hg}$ behandelt wurde, wo ich aber gleichwohl im Jahre 1885, also nach 16 Jahren, eine grosse Menge $\mathrm{Hg}$ im Harne fand. Hätte ich mich hier mit den Aufschlüssen begnügt, die das Journal des Krankenhauses lieferte, würde ich zu dem unnatürlichsten Ergebniss gelangt sein. Nähere Nachforschungen ergaben auch, dass die Patientin im Laufe des letzten Jahres mehrere Male eine Menge $\mathrm{Hg}$ gegen Ungeziefer in die Pubes eingerieben hatte und dass dieses das letzte Mal erst einige Tage vor der Untersuchung geschehen war. ${ }^{1}$ ) Ihre Hg-Elimination war nun leicht zu erklären, und ich hatte es nicht nöthig, einen so merkwürdigen Casus zu annotiren, der mit meinen übrigen Untersuchungen ganz im Streite gestanden haben würde. Wenn man weiss, wie leicht $\mathrm{Hg}$ bei Einreibung ${ }^{1}$ ) von Mercurialsalbe in die Pubes absorbirt wird, und wenn man weiss, wie oft solche Salbe eingerieben wird - wenn man

1) Anm. lch wusste damals nicht, dass es das verdunstete und nicht das eingeriebene Quecksilber ist, welches absorbirt wird. 
weiter weiss, in wie grosser Menge $\mathrm{Hg}$ durch die Abscesshöhlen, Geschwüre u. s. w. absorbirt wird, und wenn man weiss, dass Sublimat und wie oft solches heut zu Tage in der chirurgischen Praxis angewendet wird, und wenn man ferner weiss, dass bei der Einreibungsprocedur Personen, die in der Nähe sitzen, $\mathrm{Hg}$ in den Körper bekommen können - so liegt es nahe, zu argwöhnen, dass in den Fällen, wo sich z. B. 12, 13 Jahre nach abgeschlossener $\mathrm{Hg}-B$ ehandlung $\mathrm{Hg}$ im Harn nachweisen lässt, von den betreffenden Personen später in der einen oder anderen Weise $\mathrm{Hg}$ angewendet worden ist und also das im Harn gefundene Quecksilber aus einer viel späteren Zeit als der herstammt, wo diese Personen das letzte Mal eine ordentliche Quecksilberbehandlung gegen Syphilis durchgemacht haben. So lange man diese Möglichkeit nicht bestimmt ausschliessen kann, liefern diese Fälle keinen Beweis dafür, dass das Quecksilber so und so viel Jahre im Körper remaniren kanm. “ ${ }_{n}$ Bei allen Untersuchungen, die ich in Betreff dieser Frage ausgefiuhrt, habe ich auch in jeder Weise versucht, von den Patienten zu erfahren, ob sie seit der letzten ordentlichen $\mathrm{Hg}$-Behandlung $\mathrm{Hg}$ in irgend einer Weise angewendet haben. Besonders ist dieses bei den Prostituirten nothwendig gewesen, die oft mit Pediculi pubis behaftet sind und gegen dieselben gewöhnlich Mercurialsalbe anwenden. Aber auch ein anderer Umstand trägt dazu bei, dass man von den Prostituirten, wenn man nicht Acht gibt, irre geführt werden kann. Es ist ibnen nämlich verboten, sich wegen venerischen Krankheiten von privaten Aerzten behandeln zu lassen, sondern es soll dieses im Krankenhause geschehen. Es dürfte daher anzunehmen sein, dass das dort geführte Journal zuverlässige Angaben über die Zeit liefern müsse, wo eine solche Frau das letzte $\mathrm{Mal} \mathrm{Hg}$ erhalten hat. Dieses ist indessen nicht der Fall. Mehr als eine dieser Prostituirten sucht sich der Gesundheitsbesichtigung zu entziehen, indem sie, sobald sie glaubt, krank zu sein, vom Orte abreist u. s. w., und mitunter gelingt es ihr dann, während der Zeit eine mehr oder weniger ordentliche Hg-Behandlung durchzumachen. Aus Furcht vor der Strafe sucht sie dieses zu verheimlichen, und wenn man dann bei der Untersuchung ihres Harnes eine grössere oder kleinere Menge $\mathrm{Hg}$ in dem- 
selben findet, könnte man ja versucht sein zu glauben, dass. dieses $\mathrm{Hg}$ seit der letzten im Krankenhause durchgemachten Quecksilbercur im Organismus remanirt. Nur durch meine bestimmte Behauptung, dass $\mathrm{Hg}$ ausserhalb des Krankenhauses angewendet worden ist, habe ich das eine oder andere Mal eine Prostituirte dazu gebracht, dieses einzugestehen, und dadurch bin. ich der Nothwendigkeit entgangen, eine unrichtige Angabe aufzuzeichnen."

„Zahlreiche Fälle könnte ich anführen, um zu zeigen, mit wie grosser Vorsichtigkeit man zu Wege gehen muss, wenn man hinsichtlich der Remanenz des Quecksilbers im Organismus einige Schlüsse ziehen will. So weit es mir möglich gewesen ist, habe ich auch bei meinen Untersuchungen alle Vorsicht beobachtet, doch kann ich natïrlicherweise nicht behaupten, dass alle Angaben, die ich erhalten, richtig und also meine Untersuchungen in dieser Frage völlig zuverlässig sind."

Nachdem ich hierauf im Detail über alle meine hierhergehörigen Untersuchungen berichtet, sage ich: „Dass die vorstehenden Angaben, so weit es sich um meine privaten Patienten handelt, richtig sind, davon bin ich völlig überzeugt; dagegen finden sich unter den Prostituirten einige Fälle, die Zweifel an der Wahrheit der gelieferten Angaben erwecken müssen, und es ist zu vermuthen, dass die betreffenden Prostituirten, seit sie im Krankenhause das letzte Mal gegen Syphilis behandelt worden sind, Quecksilber angewendet haben." "Ich habe deshalb in der folgenden Tabelle die Prostituirten und meine privaten Patienten gesondert angefübrt."

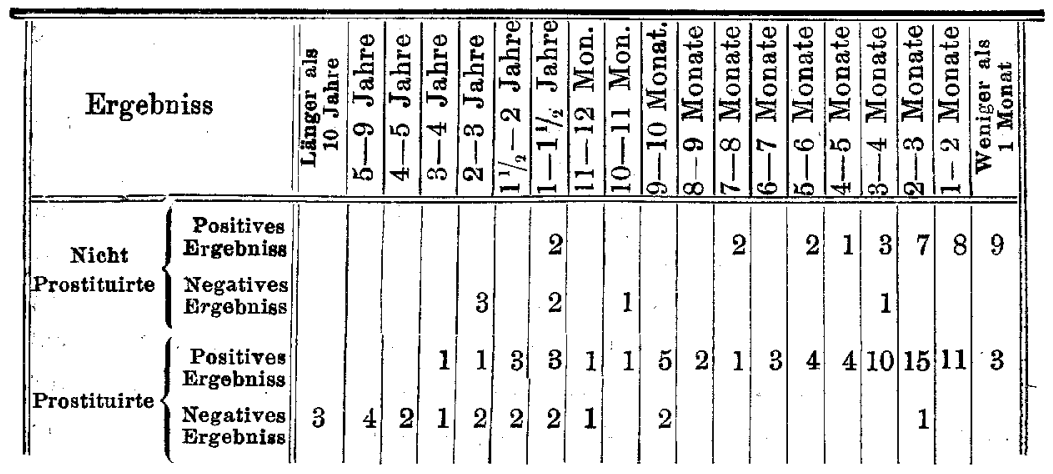


"Hätte man das Recht, nach den bei Prostituirten ausgeführten Untersuchungen zu urtheilen, würde das Quecksilber in der Mehrzahl der Fälle das ganze erste Jahr nach einer ordentlichen Behandlung mit $\mathrm{Hg}$ im Organismus remaniren; aber aus Gründen, die. ich oben angeführt habe, liefern diese Untersuchungen keine vollgiltigen : Beweise."

„Dass sich Hg gleichwohl so lange wie sieben Monate nach der Behandlung mit Quecksilber im Organismus finden kann, davon bin ich überzeugt, da ich im Harn zweier privaten Patienten noch nach so langer Zeit nach der letzten Behandlung mit Hg Quecksilber gefunden habe; diese Patienten sind völlig zuverlässige Personen, und sie verneinen es bestimmt, in der genannten Zeit in irgend einer Weise mit $\mathrm{Hg}$ zu thun gehabt zu haben. Daisselbe ist auch mit zwei anderen Patienten der Fall, bei denen ich nach mehr als einem Jahre nach abgeschlossener Hg-Cur Quecksilber im Harn gefunden habe. In dem einen Falle untersuchte ich den Patienten 10 Monate und 18 Tage, 11 Monate und 11 Tage und $1 \mathrm{Jahr}$ und 29 Tage nach dem Abschluss der Behandlung mit $\mathrm{Hg}$ und fand bei ihm jedes Mal Quecksilber im Harn, obschon nur in sehr geringer Menge. In dem anderen Falle hatte der Patient vor mehr als 1 Jahre 50 Einreibungen bekommen. Er hatte später Jodcalium genommen, doch verneint er, dass etwas $\mathrm{Hg}$ zugesetzt war. Diese beiden Fälle sprechen wenigstens für die Möglichkeit, dass Hg nach Jahr und Tag nach der Behandlung mit Quecksilber noch im Organismus remaniren kann. Dass viele Monate vergehen, ehe es vollständig eliminirt ist, ist im höchsten Grade wahrscheinlich, und dass es in der Regel 4-6 Monate nach beendigter Behandlung im Körper remanirt, scheint mir völlig sicher zu sein."

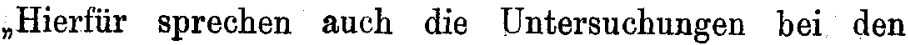
Prostituirten. Von 48 Fällen, welche hier unnerhalb der ersten 6 Monate nach Abschluss der Behandlung untersucht worden sind, war das Ergebniss nur in einem einzigen Falle negativ, und auch von 16 zwischen 6 und 12 Monaten nach beendigter Hg-Behandlung untersuchten Fällen sehen wir ein negatives Ergebniss nur in 3 Fällen. Dann zeigt sich aber ein positives Ergebniss nur in 6 von 10 zwischen 1 und 2 Jahren nach Ab- 
schluss der Hg-Behandlung untersuchten Fällen, ausserdem in 1 Falle, der nach mehr als 2 Jahren, und in 1 Falle, der nach mehr als 3 Jahren, nachdem die Patientin das letzte Mal Hg erhalten haben soll, untersucht worden ist. Diese Fälle sind natürlich im höchsten Grade zweifelhaft. Dass diese Patienten in den letzten Wochen oder Monaten vor der Untersuchung keine wirkliche $\mathrm{Hg}$-Behandlung erhalten haben, hat man allen Grund anzunehmen, da die Quecksilbermenge, die sich in ihrem Harne fand, nur sehr gering war. Von drei Fällen findet sich aufgezeichnet: ein paar sehr kleine $\mathrm{Hg}$.Kügelchen, von einem anderen: ein par kleine $\mathrm{Hg}$-Kügelchen, und von zweien: einige kleine $\mathrm{Hg}$-Kügelchen. Nur yon einem Falle ist aufgezeichnet: einige nicht kleine Hg-Kügelchen, aber in diesem Falle hatte die Patientin syphilitische Ulcerationen im Halse, und es ist daher möglich, dass sie sich vor ibrer Aufnahme in das Krankenhaus $\mathrm{Hg}$ verschafft hatte. In den übrigen Fällen waren die Patientinen mit Ausnahme einer einzigen alle wegen weichen Chancre in das Krankenhaus aufgenommen worden, daher sich kein Grund zu der Annahme findet, dass sie in der letzten Zeit ausserhalb des Krankenhauses eine $\mathrm{Hg}$-Behandlung begonnen hatten, sondern es ist das $\mathrm{Hg}$ wahrscheinlich in einer anderen Weise, z. B. durch Einreibung von Mercurialsalbe in die Pubes, was die Frauen sich oft schämen zu erzählen, in den Organismus gekommen."

Es geht ja aus diesen Untersuchungen, wie ich gefunden, deutlich hervor, dass das $\mathrm{Hg}$ zwar nicht Jahre, aber doch Monate nach Abschluss einer $\mathrm{Hg}$-Behandlung constant im Harn des Patienten nachgewiesen werden kann, mit anderen Worten, dass es so lange im Organismus des Patienten remanirt. Aber es ist nicht dieses, was in praktischer therapeutischer Hinsicht von Gewicht ist; von Gewicht ist es zu wissen, wie lange nach einer Behandlung mit $\mathrm{Hg}$ unter der oder der Form so viel $\mathrm{Hg}$ im Körper zurückbleibt, dass es sich berechnen lässt, ob das im Körper remanirende $\mathrm{Hg}$ therapeutisch oder, wenn man es so nennen will, prophylaktisch wirken kann, d. h. dem Auftreten eines allgemeinen Recidives vorbeugen kann (denn das Auftreten eines vereinzelten Symptomes an einer irritirten Stelle, wie z. B. der Vulva, den Lippen u. s. w., 
kann die grösste im Körper remanirende $\mathrm{Hg}$-Menge nicht immer verhindern). Für die Untersuchungen, die ich in dieser Hinsicht im Jahre 1885 ausgeführt habe, will ich aus meinem Aufsatz darüber vom Jahre 1886 folgende Tabelle anführen, welche die approximativen Mengen des eliminirten $\mathrm{Hg}$ zu verschiedenen Zeitperioden nach Abschluss der Behandlung angibt.

Ich habe diese approximative Berechnung der Hg-Menge auf die Anzahl und Grösse der $\mathrm{Hg}$-Kügelchen gegründet. In dem genannten Aufsatz Seite 14 und 15 habe ich ein Schema mitgetheilt, nach welchem ich die Hg-Menge als höchst bedeutend, bedeutend, gross u. s. w. bezeichne.

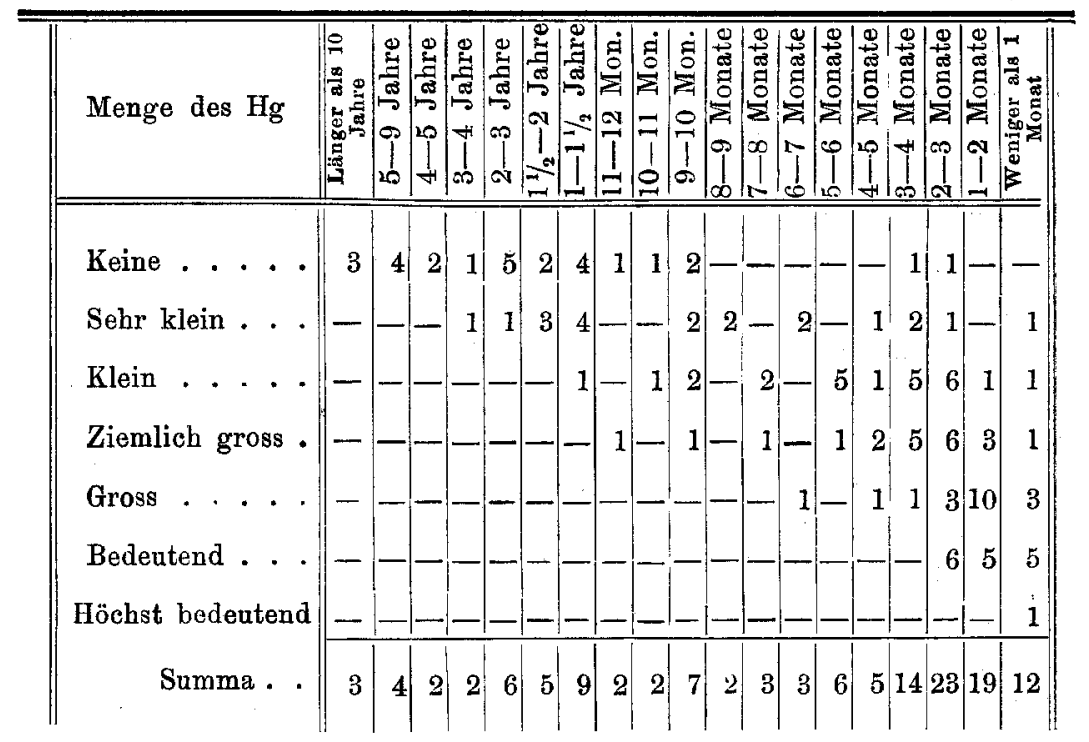

Wir ersehen aus dieser Tabelle, dass die Menge des remanirenden $\mathrm{Hg}$ sich Monat für Monat nach dem Abschluss der Behandlung vermindert (die vorhandenen Verschiedenheiten finden in einer verschieden starken Behandlung ihre Erklärung). Natürlicherweise muss eine solche Verminderung des remanirenden $\mathrm{Hg}$ auch nach der Anwendung der Behandlung mit Hg-Säckchen geschehen; da es nun in praktischer Hinsicht nicht das Geringste zu bedeuten hat, wie lange $\mathrm{Hg}$ bei der Anwendung der Hg-Säckchen und auch bei der Anwendung 
der Injection unlöslicher Salze nach Abschluss der Behandlung im Harn nachgewiesen werden kann, so habe ich mich in Betreff dieser. Behandlungsformen damit begnügt, zu ermitteln, wie lange eine grosse Menge nach Anwendung derselben im Organismus remanirt, woraus natürlicherweise nicht der Schluss gezogen werden darf, dass die Elimination von $\mathrm{Hg}$ aufhört, sobald keine grössere Menge mebr eliminirt wird." Ich hoffe, dass meine Angaben in der Regel nicht so gedeutet worden sind.

Ich will nun zu einer Anmerkung oder, richtiger gesagt, zu einem Schlusse übergehen, den Gschwend aus seinen genauen Untersuchungen über die therapeutische Wirkung der Hg-Säckchen zieht - ein Schluss, den ich durchaus nicht als berechtigt ansehen kann.

Wie ich bereits erwähnt habe, ist Gschwend der Ansicht, dass bei der Anwendung der Hg-Säckchen Recidive ungewöhnlich rasch auftreten, und er sagt nun: „Dieses rasche Recidiviren lässt darauf schliessen, dass die Quecksilberremanenz in diesen Fällen nur unbedeutend gewesen sein kann."

Ich habe zwar schon friher gezeigt, dass die Elimination des $\mathrm{Hg}$ bei Anwendung dieser Behandlungsform nicht schneller als bei der Anwendung der meisten anderen Behandlungsmethoden geschieht; da ich es aber stets zur Gewohnheit gehabt habe, zu untersuchen, ob eine gegen meine Angaben erhobene Anmerkung richtig ist, so habe ich dieses auch hier gethan. Es ist mir da gleichwohl als zweckmässiger und richtiger erschienen, hierfür wirkliche Untersuchungen über die Remanenz des Quecksilbers anzuführen, als, wie Gschwend, zu versuchen, sie in indirecter Weise nachzuweisen.

Ich will nun über die Remanenz des Quecksilbers in 22 Fällen berichten, die ich, bis auf zwei, aller meiner privaten Praxis entnommen habe, damit ich richtig sicher sein kann, dass von den Patienten nicht extra $\mathrm{Hg}$ angewendet worden ist; dass dieses auch in den beiden Fällen (Nr. 11 und 17 der Tabelle), die im Krankenhause behandelt worden sind, nicht geschehen ist, davon glaube ich überzeugt sein zu können. Ich habe in der folgenden Tabelle die Dauer der Behandlung, die Anzahl der Tage nach Abschluss derselben und die Menge 
des gefundenen $\mathrm{Hg}$, approximativ berechnet, angegeben. In Bezug auf die Dauer der Behandlung will ich hier bemerken, dass ich stets, welche Behandlungsweise ich auch anwende, eine kräftige Behandlung gebe. Liegt ganz frische Syphilis (die erste Bebandlung) vor oder sind die Symptome schwer, so gebe ich, so weit dieses möglich ist, eine sehr kräftige und lange andauernde Behandlung. Eine mehr als dreissigjährige Erfahrung als Syphilidologe hat mich gelehrt, dass eine $\mathrm{Hg}$ Behandlung, wenn mit derselben ein wirklich gutes Resultat erzielt werden soll, kräftig sein muss und eine nicht zu kurze Dauer haben darf.

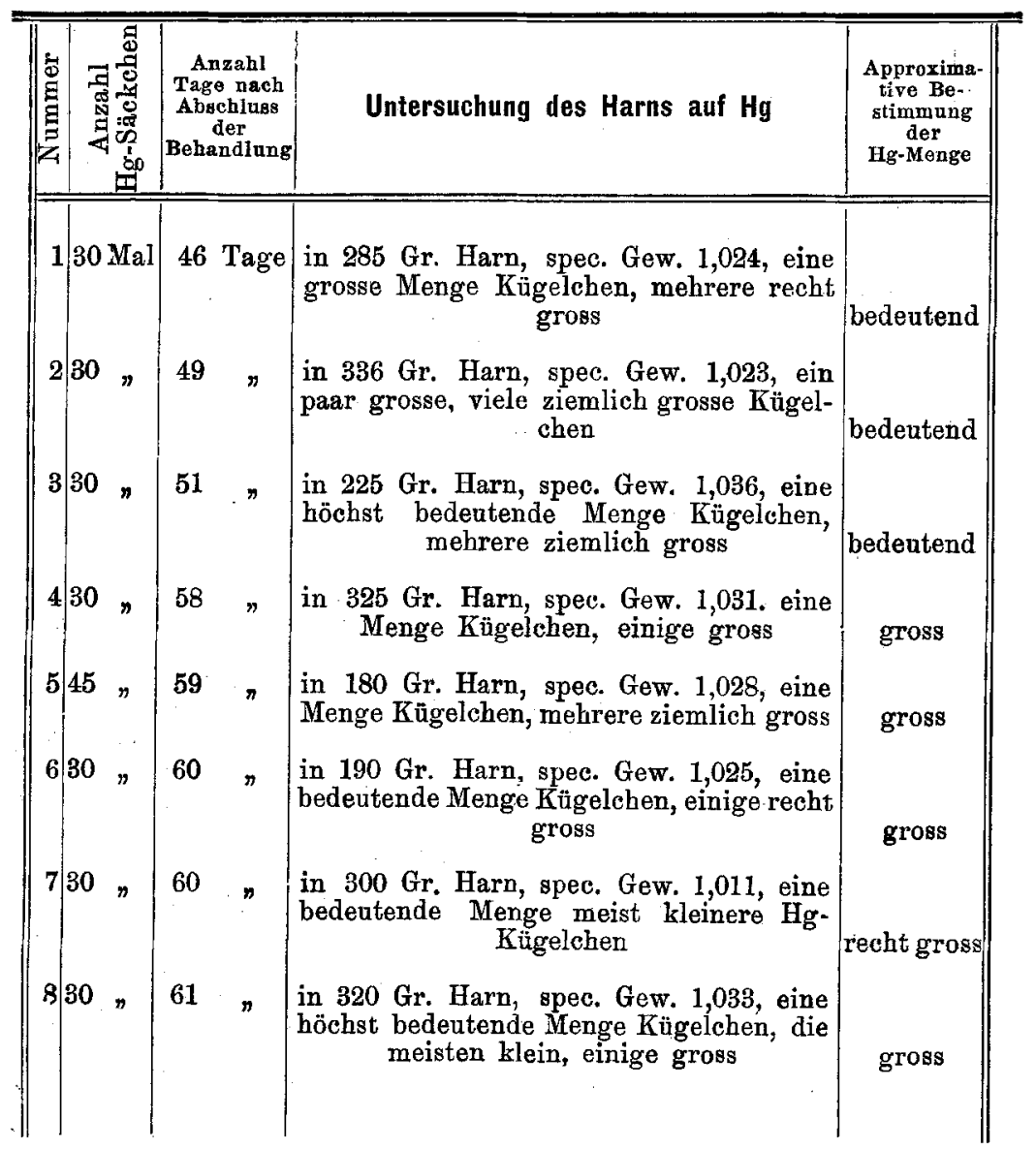




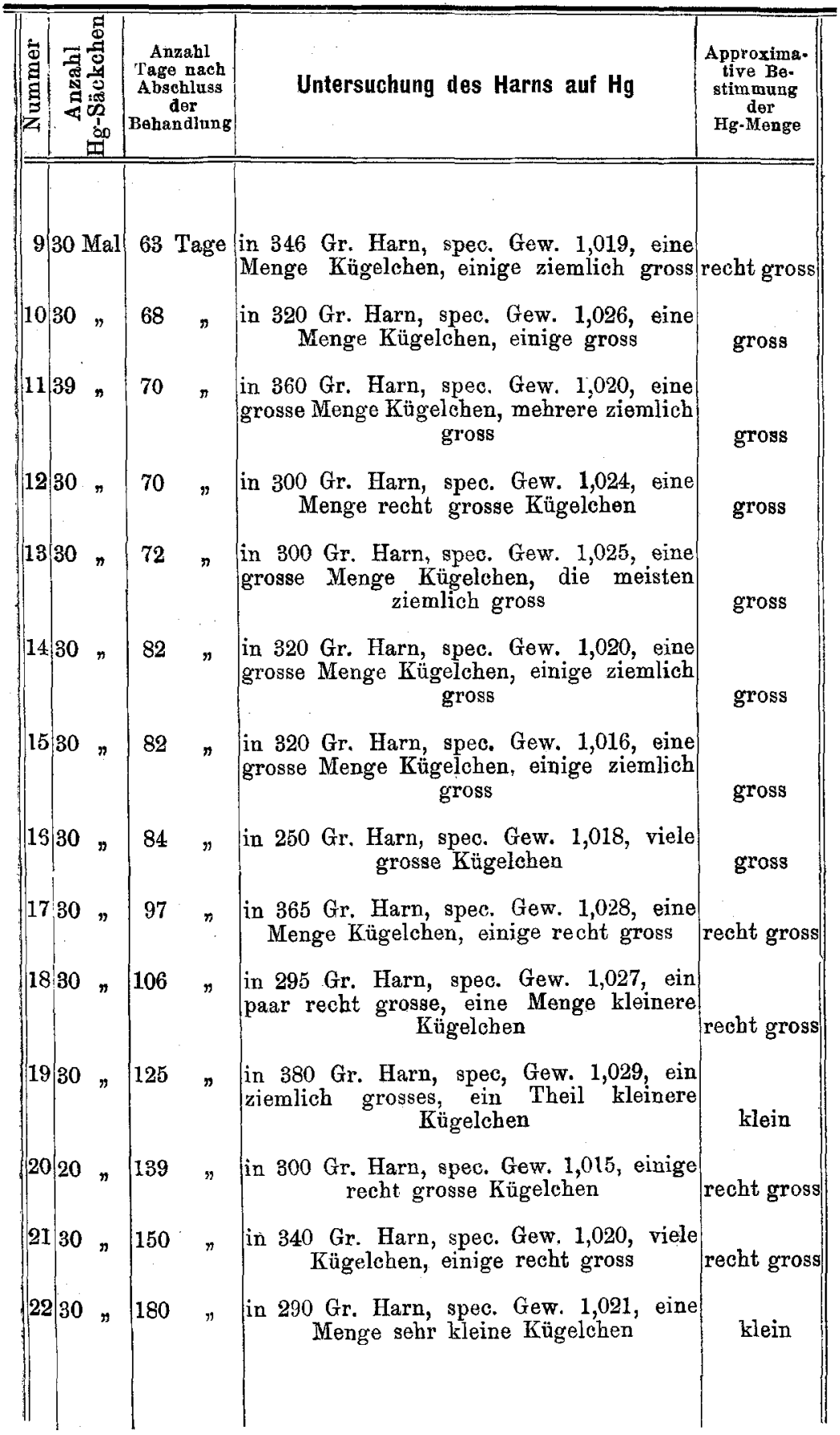


Wir ersehen aus der Zusammenstellung, dass sich die Menge des eliminirten Quecksilbers, die ja die Menge des im Organismus remanirenden Quecksilbers angibt, constant in demselben Verhältniss vermindert, in welchen die Zeit nach dem Abschluss der Behandlung sich vermehrt. Schon im Jahre 1886 habe ich darauf hingewiese, dass man bei der approximativen Berechnung der Menge des eliminirten $\mathrm{Hg}$ die Menge des untersuchten Harnes und das spec. Gewicht desselben berücksichtigen muss. Es ist ja klar, dass z. B. in $350 \mathrm{Gr}$. von gleicher Beschaffenheit eine grössere Menge Hg nachzuweisen sein muss, als in $200 \mathrm{Gr}$. Deshalb kann ich mit Grund behaupten, dass, wenn in den Fällen 5 und 6 eine gleich grosse Menge Harn untersucht worden wäre, wie in den übrigen Fällen, also ungefähr $325 \mathrm{Gr}$, ganz sicher in ihnen auch die Menge des eliminirten $\mathrm{Hg}$ als bedeutend, anstatt als gross hätte bezeichnet werden können; das niedrige specifische Gewicht in Fall 7 kann sehr gut die Ursache sein, dass die Menge des eliminirten $\mathrm{Hg}$ hier nur als recht gross (anstatt gross) bezeichnet werden konnte.

Vergleichen wir indessen diese Tabelle mit Tabelle II, in welcher sich eine Menge mit Einreibungen behandelte Fälle aufgenommen finden, so zeigt sich zwischen diesen Tabellen eine in die Augen fallende Uebereinstimmung. - Diese wirklichen Untersuchungen auf $\mathrm{Hg}$ zeigen also, dass das Quecksilber bei der Behandlung mit Hg-Säckchen nicht so schnell eliminirt wird, wie man glaublich zu machen gesucht hat, und dass die Methode nicht mit einem solchen Fehler behaftet ist.

Ich will hier bemerken, dass in die Tabelle II kein Fall von Injectionen unlöslicher Salze aufgenommen ist; ich hatte damals noch nicht damit begonnen, solche Injectionen zu geben. Bei diesen Injectionen findet nämlich eine länger andauernde und kräftigere Elimination von $\mathrm{Hg}$ statt, da bei ihnen auch nach Abschluss der Behandlung eine Absorption von $\mathrm{Hg}$ von den Depots aus stattfindet, die durch die Injectionen angelegt worden sind; dieses gilt in einem noch höheren Grade von Injectionen von Langs Ol. cinerum. Ich habe auch hervorgehoben, dass die Injectionen unlöslicher Salze sowohl in dieser, wie in vielen anderen Hinsichten eine Idealbehandlung 
wären, wenn sie nicht so oft diese schmerzhaften Infiltrationen hervorriefen, die ihre Anwendung in einer Menge von Fällen so beschwerlich machen.

Können nun diese Worte über Quecksilberdepots auch in Bezug auf die wirkliche Einreibungscur angewendet werden? Gschwend scheint dieses anzunehmen, denn er sagt: „dass sich bei der Einreibungscur ein gewisses Depot von Quecksilber in den Poren der Talg- und Schweissdrüsen festsetzt und nach Charles Mauriac eine ebenso beständige und milde Absorption durch die Haut erleidet, darum die längere Remanenz und das spätere Auftreten von Recidiven, während bei der Säckchenbehandlung diese Depots fehlen und daher die Quecksilberzufuhr mit dem Aussetzen des Säckchens aufhört.“

Ja! so haben Mauriac und G schwe nd gesagt, aber haben sie auch für die Richtigkeit ihrer Aeusserung einen Beweis beigebracht? Mir wissentlich haben sie keine Untersuchungen ausgeführt, um das, was sie hier gesagt, zu beweisen. Aber finden sich andere Untersuchungen, die dafür vielleicht eine Stütze sein können? Wir wissen z. B.. dass Neumann und $F \ddot{u} h r b r i n g e r$ vor langer Zeit dargethan haben, dass bei Einreibungen $\mathrm{Hg}$-Salbe in die Haarfollikeln und die Talgdrüsen kommt. Manassein's und namentlich Brock's Untersuchungen haben bekräftigt, dass dieses der Fall ist und dass bei Einreibungen $\mathrm{Hg}$ ziemlich tief in diese Mündungen eindringt. Brock sagt: „Die Quecksilberkügelchen lagen zwischen den Zellen der obersten Harnschichtabschnitte, in den Haarfollikeln zwischen Haar und einer Wurzelscheide, nach unten zu etwa bis zur unteren Grenze des oberen Drittels, ferner in den Müindungen der Talg- und Knäueldrüsen." Von bier wird aber das $\mathrm{Hg}$ nicht absorbirt, sondern "in dem Masse, wie die Hornschicht sich abstösst, wird das Metall gleichfalls von der Oberfläche entfernt; das wachsende. Haar nimmt bei der Einreibung mit der Haarwurzel verklebten Salbenmassen aus der Tiefe mit sich fort." Brock sagt ferner: nach 14 Tagen "fanden sich Quecksilberkügelchen nur ganz vereinzelt, und zwar in den allerobersten Partien der Hornschicht; die Talgdrüsen- und die Knäueldrüsen-Mündungen sind frei von Quecksilberkügelchen; in den Haarfollikeln finden sich dieselben nur an der Mündung rings .um das Haar herum." 
Es unterliegt also keinem Zweifel, dass sich hier in der ersten Zeit nach einer Einreibungscur eine Art von Quecksilberdepots in der Haut finden. Davon kann man sich auch in anderer Weise leicht überzeugen. Wenn man wirkliche Einreibungen macht und die Salbe die Nacht über aufgestrichen auf der Haut liegen lässt und sie erst am Morgen darauf abwäscht, so kann man schon mit dem blossen Auge, deutlicher aber noch mit der Lupe, in der ersten Zeit nach den Einreibungen in den Mündungen der Haarfollikeln, namentlich in den grösseren, schwarze Punkte sehen, die anfangs in gleicher Höhe mit der Haut liegen, sich aber nachher über sie erheben. Wenn man diese schwarze Masse vorsichtig aus der Mündung herauspresst, sie in eine feine Glasröhre bringt und die Röhre dann an der Stelle, wo die Masse liegt, erhitzt, so kann man nachher mit dem Mikroskope sehen, dass eine Menge $\mathrm{Hg}$-Kügelchen sublimirt worden sind. Solche Hg-Anhäufungen habe ich in den beiden Fällen, die ich näher untersucht, hauptsächlich an den Unterschenkeln, weniger zahlreich an den Oberschenkeln und auf dem Rücken und am wenigsten zahlreich an den Armen gefunden. Man findet also, sowohl makro- wie mikroskopisch. Hg-Depots in der Haut, und wenn auch der Organismus dieses $\mathrm{Hg}$ nicht durch die Haut zu absorbiren vermag, so liegt doch die Annahme nahe zur Hand, dass von diesen Depots aus $\mathrm{Hg}$ verdünsten und solchergestalt, unter der Form von $\mathrm{Hg}$-Dämpfen, absorbirt werden kann.

Aber unter welcher Form findet sich nun das $\mathrm{Hg}$ in diesen Follikelmündungen? Findet es sich in ihnen unter der Form von metallischem Quecksilber, so ist die Möglichkeit vorhanden, dass es verdünsten und absorbirt werden kann; andernfalls ist es $\mathrm{Z}_{\text {weifel }}$ unterworfen, ob es verdünsten und absorbirt werden kann.

Ich habe folgende zwei Versuche ausgeführt, theils um zu erfahren, unter welcher Form das $\mathrm{Hg}$ in den Follikelmündungen findet, theils um zu sehen, wie lange es dort remanirt.

Fall VIII. Th. B. Den 31./V. wurde um $8 \mathrm{Uhr}$ Nachm. an beiden Waden eine ordentliche, halbstündige Einreibung von $6 \mathrm{Gr}$. Ung. Hydr. (1: 2) ausgeführt und dabei, wie gewöhnlich, nicht alles Hg eingerieben, sondern ein Theil der Salbe aufgestrichen auf der Haut liegen gelassen; unmittelbar nach der Einreibung wurde (der Reinlichkeit wegen) ein 
Welander.

Leinentuch um jede Wade gebunden. Nach 12 Stunden wurde die auf der Haut liegende Salbe abgewaschen. Den 1./VI. wurde dieselbe Procedur an dep Oberschenkeln, den 2./VI. auf dem Rücken and den 3./VI. an den Armen ausgeführt. Den 4./VI. wurde die zuletzt eingeriebene (aufgestrichene) Salbe abgewaschen und mit den Einreibungen aufgebört. Den 7./VI. bekam die Patientin ein allgemeines Bad. Den 11./VI. zeigen sich an den Unterschenkeln in vielen grossen Follikelmündungen schwarze Pfropfen, von denen sich mehrere etwas über die Fläche der Hant erheben; an den Oberschenkeln finden sich hier und da solche Pfropfen, aber auf dem Rücken und an den Armen zeigt sich nur der eine und der andere. Ein kleiner solcher schwarzer Pfropfen wurde aus der Follikelmündung herausgenommen, in eine feine Glasröhre gebracht und über einer Gasflamme erhitzt, wobei $\mathrm{Hg}$ sublimirte und sich unter dem Mikroskop eine Menge Hg-Kügelchen zeigten. Den 15./VI. zeigen sich auf dem Rücken und an den Armen keine schwarzen Pfropfen mehr, an den Oberschenkeln kommen sie nur noch vereinzelt vor; aber an den Unterschenkeln sind sie noch vorhanden; es zeigt sich die Mehrzahl derselben über die Hautfläche erhaben; an vielen Stellen sitzt ein solcher Pfropfen an dem Haare sogar etwas oberhalb der Hautfläche. Viele der Pfropfen haben ein grauliches Aussehen. Einige solche Pfropfen wurden in Glycerin auf ein Objectglas leicht angedrückt, wo sich unter dem Mikroskop von epidermisähnlichen, ganz sicher aus der Follikelmündung stammender Zellen umgebene Agglomerate von $\mathrm{Hg}$-Kügelchen (?) zeigten; zwischen den Epidermisschuppen konnten Hg-Kügelchen nicht mit Sicherheit nachgewiesen werden. Den 18./VI. bekam die Patientin ein Bad; nur an den Unterschenkeln zeigen sich noch solche schwarze Pfropfen, doch an Zahl vermindert. Den 19./VI. in solchen Pfropfen Anhäufungen von glänzenden Hg-Kügelchen (?).

Fall IX. L. A. Wirkliche Einreibungen wurden in derselben Weise wie im vorigen Fall den 8./VI. an den Waden, den 9./VI. an den Oberschenkeln, den 10./VI. am Rücken und den 11./VI. an den Armen gemacht. Den 12./VI. zeigen sich an allen Stellen, wo die Einreibung ausgeführt worden ist, schwarze Punkte in der Haut, und zwar an den Unterschenkeln in grosser Menge, ebenso auch am Rücken, an den Oberschenkeln und den Armen aber in geringerer Menge; einer dieser Pfropfen wurde herausgenommen und in einer Glasröhre erhitzt, wobei eine Menge $\mathrm{Hg}$-Kügelchen sublimirten. Den 15./VI. fangen an den Unterschenkeln, namentlich dem rechten, die Pfropfen an, sich über die Hautfläche zu erheben; an den übrigen Stellen zeigen sie sich an Zahl vermindert; ein paar Pfropfen wurden mikroskopisch in Glycerin untersucht und gaben dabei ganz dasselbe Bild wie im vorigen Falle. Den 18./VI. badete die Patientin. An den Armen, dem Rücken und den Oberschenkeln vereinzelte Pfropfen; an den Waden, insonderheit der linken, die Anzahl der Pfropfen vermindert und die Mehrzahl derselben etwas über die Hantfläche erhaben, ein Theil von ihnen sogar ganz über dieselbe herausgeschoben. Jen 23./VI. bei mikroskopischer Untersuchung der Pfropfen 
von verschiedenen Stellen sowohl vereinzelte Hg-Kügelchen, wie Anhäufungen von glänzenden solchen Kügelchen (?) zu sehen.

Es schien mir durch diese Untersuchungen dargethan zu sein, dass das Quecksilber unter der Form von metallischem $\mathrm{Hg}$ in der Haut remanirt; fortgesetzte Untersuchungen zeigten aber, wie schwer es ist, kleine $\mathrm{Hg}$-Kügelchen mit Sicherheit von kleinen Luftbläschen zu unterscheiden, die stets bei diesen Untersuchungen auftreten. Dieses zeigte sich noch deutlicher, als ich in einigen Fällen versuchte, an eingeriebenen Stellen die Epidermis abzuschaben, um zu sehen, ob sich zwischen den Epidermisschuppen Hg-Kügelchen fanden. Bei allen Personen, die ich untersuchte, ja sogar bei denen, die kein $\mathrm{Hg}$ erhalten hatten, traten kleine Luftbläschen auf, die kleinen $\mathrm{Hg}$-Kügelchen täuschend ähnlich waren. Ich kann deshalb aus den beiden ebenerwähnten Fällen hinsichtlich der Form der Hg-Remanenz keine Schlüsse ziehen.

Um indessen zu erfahren, ob $\mathrm{Hg}$ nach seiner Einreibung in nennenswerther Menge zwischen den Epidermiszellen remanirt, habe ich eine Anzahl Versuche in der Weise ausgeführt, dass ich Epidermisschuppen an Stellen abschabte, wo zwar Finreibungen geschehen waren, sich aber mit der Lupe keine schwarzen Pfröpfchen in den Follikelmündungen entdecken liessen, das Abgeschabte nachher in feine Glasröhren brachte, es hier erhitzte und dann untersuchte, ob sich einige $\mathrm{Hg}$ Kügelchen sublimirt zeigten. Ich habe in dieser Weise niemals $\mathrm{Hg}$ nachzuweisen vermocht, selbst nicht einmal da, wo ich, wie in dem folgenden Fall, diese Untersuchung zwei Tage nach der Einreibung des $\mathrm{Hg}$ ausführte.

Fall X. K. A. Den 21./VII. Einreibung an den Waden, Bad den 22./VII. und den 23./VII. Den 23./VII. wurden an den Waden an einer Menge Stellen, wo schwarze Pfröpfchen in den Follikelmündungen nicht zu entdecken waren, Epidermisschuppen abgeschabt, in feine Glasröhren gelegt und erhitzt, keine Hg-Kügelchen liessen sich entdecken. Unmittelbar hierauf wurden zwei kleine schwarze Pfröpfchen aus den Follikelmündungen gehoben, in feine Glasröhren gebracht und erhitzt - hierbei liessen sich mikroskopisch eine Menge $\mathrm{Hg}-\mathrm{Kügelchen}$ nachweisen.

Zwischen den Epidermiszellen habe ich also nie mit Sicherheit $\mathrm{Hg}$ nachweisen können, wohl aber konstant in den kleinen schwarzen Pröpfchen in den Follikelmündungen.

Wie lange nun solche $\mathrm{Hg}-\mathrm{Pfröpfchen} \mathrm{sich} \mathrm{in} \mathrm{den} \mathrm{Follikel-}$ 
mündungen finden kömnen, scheint ganz sicher darauf zu beruhen, wie oft der Patient badet und an den eingeriebenen Stellen gebürstet wird; wie lange sich das $\mathrm{Hg}$ in diesen Pröpfchen erhält, vermag ich nicht anzugeben, doch habe ich in ihnen noch nach 3 Wochen $\mathrm{Hg}$ nachweisen können.

Diese Versuche haben zwar keinen Aufschluss über die Form geben können, unter welcher das $\mathrm{Hg}$ in der Haut remanirt, doch haben sie auf alle Fälle sicher gezeigt, dass eingeriebenes $\mathrm{Hg}$ wirklich eine Zeit lang in der Haut remanirt, womit die Möglichkeit der Verdünstung und Absorption desselben gegeben ist.

Hat nun eine solche wirklich stattgefunden? und wenn dieses der Fall gewesen ist: kann sie so gross gewesen sein, dass sie eine praktische Bedeutung gehabt hat? Wir können uns ja eine Verdünstung theils von diesen Pfropfen, aber theils auch von dem Quecksilber denken, das sich nach Brock zwischen den Zellen der obersten Hornschichtabschnitte abgelagert findet. In demselben Verhältniss, in welchem dieser abgestossen wird, wird auch das Quecksilber abgestossen. Da nun Brock Hg-Kügelchen nach 14 Tagen nur ganz vereinzelt in den obersten Partien der Hornschicht finden konnte, und da es mir nicht geglïckt ist, bei der Erhitzung abgeschabter Epidermis sublimirtes $\mathrm{Hg}$ nachzuweisen, ist es sehr wahrscheinlich, dass die Verdünstung von diesem remanirenden $\mathrm{Hg}$, von diesen $\mathrm{Hg}$-Depots nicht gross sein kann. In den Haarfollikeln konnte ja B rock nach 14 Tagen $\mathrm{Hg}$-Kügelchen rings um das Haar herum sehen, was ja gut mit meinen Untersuchungen der herausgepressten schwarzen Pfröpfchen übereinstimmt; von diesen liesse es sich ja denken, dass von ihnen aus eine grössere Verdünstung und Absorption von $\mathrm{Hg}$ geschehen könnte. Aber à priori ist dieses nicht wabrscheinlich. Wohl konnte man eine Menge Follikeln an den Unterschenkeln mit schwarzer Hg-Masse gefüllt sehen, die Quecksilber enthielt, aber die Grösse der Verdünstung ist ja von der Verdünstungsfläche des Quecksilbers abhängig. Rechnet man nun auch den Flächeninhalt dieser kleinen Pfröpfchen zusammen, so ist die Verdünstungsfläche doch gleichwohl sehr klein, daher man nicht das Recht hat, hier eine grössere Verdünstung zu erwarten. 
Um dieses einigermassen zu prüfen, habe ich in diesen beiden Fällen, VIII und IX, folgende Versuche ausgeführt.

T. B. Nachdem sie den 4./VI. alle 4 Einreibungen (+ Ueberstreichung) erhalten hatte, wobei so viel $\mathrm{Hg}$ absorbirt wurde, dass sich den 8./VI. in $360 \mathrm{Gr}$. Harn, spec. Gew. 1.019, eine Menge Hg-Kügelchen fanden, von denen einige ziemlich gross waren, liess ich sie die folgenden Nächte auf der Brust eine Schachtel mit Kupferdrähten tragen. Die Möglichkeit fand sich ja für das Quecksilber, sowohl von den Follikelmündungen ans, wie von dem zwischen den Epidermiszellen abgelagerten Quecksilber zu verdünsten. Am Morgen des 5./VI. fanden sich an den Kupferdrähten der Schachtel auf der Brust viele kleine Ho-Kügelchen. Am Morgen des 6./VI. fanden sich an den Kupferdrähten der Schachtel auf der Brust eine Menge sehr kleine Hg-Kügelehea. Am Morgen des 7./VI. fanden sicb an den Kupferdrähten der Schachtel auf der Brust keine $\mathrm{Hg}$-Kügelchen. Auch an den folgenden Morgen konnten keine $\mathrm{Hg}$ Kügelchen an den Kupferdrähten entdeckt werden. Da sich an anderen Stellen als den Waden nur sehr wenig Hg-Pfropfen zeigten, machte ich einen Versuch, um zu erfahren, ob die Verdünstung dort nicht möglicherweise grösser sei, obschon die Hg-Dämpfe sich nicht bis an die Brust hinauf verbreitet haben. Ich band an jede Wade eine Schachtel mit Kupferdrähten und liess diese Schachteln die Nacht vom 16./VI. bis zum 17./VI. an diesen Stellen liegen. Am Morgen des 17. konnte an den Kupferdrähten keiner dieser Schachteln Hg entdeckt werden.

L. A. Das Ergebniss war hier vollständig dem im vorigen Falle erbaltenen gleich. Den 8./VI, Einreibung an den Waden. Den 9./VI. Einreibung an den Oberschenkeln; am Morgen liessen sich an den Kupferdrähten der Schachtel, die auf der Brust gelegen hatte, eine Menge kleine Hg-Kügelchen nachweisen. Den 10./VI. Einreibung auf dem Rücken; am Morgen des 11./VI. liessen sich eine bedeutende Menge Hg-Kügelchen nachweisen. Den 11./VI. Einreibung an den Armen. Den 12./VI. konnten eine Menge kleinere Kügelchen nachgewiesen werden (hören mit den Einreibungen auf). Den 13./VI. liessen sich an den Kupferdrähten nur einige kleine $\mathrm{Hg}-\mathrm{Kügelchen} \mathrm{nachweisen.} \mathrm{Den} \mathrm{14./VI.} \mathrm{urd} \mathrm{auch} \mathrm{an} \mathrm{den} \mathrm{folgenden}$ Morgen waren keine $\mathrm{Hg}$-Kügelchen nachzuweisen.

Hieraus könnte man ja den Schluss ziehen, dass $\mathrm{Hg}$ von diesen $\mathrm{Hg}$-Depots nicht länger als ein paar Tage verdünstet; dieses wäre aber ein unrichtiger Schluss, denn $\mathrm{Hg}$ verdünstet sicher so lange, wie diese Hg-Depots sich finden, obschon dieses nur in sehr geringer Menge geschieht. Wenn die Verhältnisse für die Verdünstung des Quecksilbers sehr günstig sind, findet man auch, dass $\mathrm{Hg}$ von diesen Depots in nachweisbarer Menge verdünstet.

Ich liess sowohl T. B. wie L. A. an Abend des 15./VI. auf denjenigen ihrer Unterschenkel, an welchem sich in den 
Follikelöffnungen die meisten schwarzen Pfropfen zeigten, unmittelbar auf die Haut eine Schachtel mit Kupferdrähten legen und darïber Wachstaffet binden, worauf der ganze Schenkel, ganz wie bei Schuster's Versuchen, ordentlich mit Gazebinden umwickelt wurde. Am Morgen darauf fand ich bei beiden Patienten an den Kupferdrähten der Schachtel ziemlich viele kleine $\mathrm{Hg}$-Kügelchen. Also ist die Verdünstung des $\mathrm{Hg}$ bei dieser höheren Temperatur, wo die Hg-Dämpfe dazu unter dem Wachstaffet concentrit sind, völlig nachweisbar. ${ }^{1}$ )

Ich führte nun Controlversuche an den beiden anderen Unterschenkeln aus, wo die Anzahl der Hg-Pfropfen kleiner war, und fand auch hier eine Hg-Verdünstung, obschon dieselbe geringer war; ja der Versuch wurde, nachdem L.A. den 18. VI. gebadet hatte, mit dem Ergebniss erneuert, dass sich auch jetzt eine $\mathrm{Hg}$-Verdünstung, obschon in geringer Menge, nachweisen liess.

Um weiter zu ermitteln, ob es hauptsächlich diese $\mathrm{Hg}$ Pfropfen in den Follikelmündungen oder das zwischen den Epidermiszellen abgelagerte Quecksilber war, von wo die Verdünstung geschah, liess ich bei L. A. sowohl an dem rechten Arm, wie an dem linken Oberschenkel, an welchen Stellen sich hier und da Hg-Pfropfen zeigten, einen solchen Wachstaffetverband mit einer Schachtel mit Kupferdräbten unmittelbar auf die Haut legen - an den Drähten beider Schachteln liessen sich einige kleine Hg-Kügelchen nachweisen. Einen solchen Versuch hatte ich am Tage vorher auch an dem rechten Oberschenkel der Patientin, wo sich kaum einige Hg-Pfropfen entdecken liessen, gemacht und hier ein negatives Ergebniss erhalten.

Ich führte einen solchen Versuch auch bei T. B. aus, und zwar vom 17./VI. bis 18./VI. an ihrem rechten Oberschenkel und vom 18./VI. bis 19./VI. an ihrem rechten Arm, an welchen Stellen sich keine $\mathrm{Hg}$ Pfropfen entdecken liessen. In keiner der Schachteln waren an den Kupferdrähten $\mathrm{Hg}$-Kügelchen nachzuweisen.

In voller Uebereinstimmung hiermit fielen auch zwei Versuche in Fall VII, F., aus, wo auf den einen Oberschenkel 5 und auf den anderen 10 Einklatschungen gegeben und dann ein Wachstaffetverband angelegt worden war. Den 16./VI. war an keinem der Schenkel ein Hg-Pfröpfehen zu entdecken. Den 16./VI. wurde der rechte, den 17./VI. der linke Ober-

1) Spätere Versuche haben dasselbe Resultat ergeben. 
schenkel umwickelt; an den Kupferdrähten keiner der auf den Schenkeln getragenen Schachteln war $\mathrm{Hg}$ nachzuweisen.

Auch wenn $\mathrm{Hg}$ im Anfange vermuthlich auch von dem $\mathrm{Hg}$ verdünstet, welches zwischen den Epidermiszellen remanirt, so ist es doch höchst wahrscheinlich, dass die geringe Verdünstung, die später geschieht, von dem $\mathrm{Hg}$ in den Follikelmündungen stattfindet.

Diese Untersuchungen zeigen uns ja unzweifelhaft, dass nach einer wirklichen Einreibungscur Quecksilber ziemlich lange in den Drüsenmündungen, namentlich da, wo dieselben, wie an den Unterschenkeln in meinen beiden Fällen, gross sind, liegen bleibt, sowie auch, dass $\mathrm{Hg}$, wenigstens ron diesen Pfropfen und anfangs wahrscheinlich auch von dem $\mathrm{Hg}$, das zwischen den Epidermiszellen remanirt, wirklich verdünsten kann. Es finden sich also auch bei der Einreibungscur remanirende $\mathrm{Hg}$-Depots. Aber können wir ihnen eine wirkliche Bedeutung in therapeutischer, prophylaktischer Hinsicht $\mathrm{zu}^{*}$ schreiben? Ich weiss nicht, wie empfindlich die von mir angewendete Probe mit den Schachteln ist, auch sind die Versuche, welche ich ausgeführt, nicht zahlreich genug, um sichere Schlüsse zu gestatten. Aber stelle ich das Ergebniss dieser Untersuchungen mit der Erfahrung zusammen, die ich bezüglich der Remanenz des Quecksilbers bei Anwendung der Einreibungscur ( + Deberstreichung), wo dieselbe sich nicht grösser und länger andauernd als bei der Anwendung der Hg-Säckchen gezeigt hat, habe, so scheint es mir nicht unberechtigt zu sein, zu behaupten, dass sich nach den Einreibungen zwar kleine $\mathrm{Hg}$-Depots in der Haut finden, von denen $\mathrm{Hg}$ verdünsten und dann vom Organismus absorbirt werden kann, dass diese Verdïnstung unter gewöhnlichen Verhältnissen aber so gering ist, dass sie nicht gern eine praktische Bedeutung haben kann. Aber auch wenn wir ihr eine etwas grössere Bedeutung zuschreiben wollten, als wir ihr, wie ich meine, zuschreiben können, so glaube ich doch, dass die Frage: kann der Vortheil, den diese geringe Menge remanirendes Quecksilber darbietet, so gross sein, dass er vollen Ersatz für die mit der Einrei. bungscur verbundenen Unannehmlichkeiten liefert, mit nein beantwortet werden muss. 
Zuletzt will ich hervorheben, dass man diese Hg-Säckchenmethode natürlicherweise nach Belieben modificiren kann, je nachdem man eine sehr kräftige, oder eine weniger kräftige Absorption von $\mathrm{Hg}$ wünscht $u$. s. w. Nicht bloss die Menge und die Stärke der Hg-Salbe, sondern auch die Grösse der Säckchen, die Zahl der Stunden, die sie täglich getragen werden, die Zahl der Tage, die sie zur Anwendung kommen u. s. w. haben offenbar Einfluss auf die Grösse der Hg-Absorption, und man kann ja, wie ich bereits 1897 hervorgehoben habe, leicht Wechselungen in der Anwendungsweise der Methode eintreten lassen, je nachdem man ein kleines, zartes Kind oder einen grossen, „intelligenten, breit gebauten" Mann zu behandeln, sehr schwere oder gelindere Symptome zu bekämpfen hat u. s. w. Dieses gilt natürlicherweise auch rom Mercuriolsäckchen und der Mercolintschürze.

Ich schloss meinen vorigen Aufsatz mit der Erklärung, dass es mir gleichgültig sei, ob meine Hg-Säckchenmethode, das Mercuriol, das Mercolint oder eine andere Methode als am zweckmässigsten befunden wird, "denn die Absicht bei meiner Arbeit ist hier, wie überall, nur die gewesen, einen Anstoss zu fortgesetzter Forschung zu geben, die uns sowohl neues Wissen schaffen, wie auch von praktischem Nutzen sein könnte." Dieses ist auch hier der Fall gewesen, und indem ich die Hoffnung ausspreche, dass diese meine unbedeutenden Untersuchungen Anstoss zu weiteren Forschungen geben werden, will ich erklären, dass ich mich den Herren Schuster und Gschwend für ihre Aufsätze zu Dank verpflichtet fühle, da dieselben der Anlass zu diesen Untersuchungen gewesen sind, welche mir zwar gezeigt haben, dass die Erklärung, die ich bezüglich des Weges der Absorption des Quecksilbers bei Anwendung der Hg-Säckchenmethode acceptirt habe, nicht ganz richtig war, welche aber doch die Richtigkeit meiner auf frühere Untersuchungen gegründeten Ansicht bestätigt haben, dass sich bei der Behandlung mit $\mathrm{Hg}$-Säckchen nicht nur eine schnelle und kräftige Absorption, sondern auch eine kräftige und lange andauernde Remanenz des Quecksilbers im Organismus findet, daher diese Methode in der von mir angegebenen Form mit Recht als eine kräftige Hg-Behandlungsmethode bezeichnet werden kann. 